Document downloaded from:

http://hdl.handle.net/10251/105879

This paper must be cited as:

Primo Arnau, AM.; Parvulescu, V.; García Gómez, H. (2017). Graphenes as Metal-Free

Catalysts with Engineered Active Sites. The Journal of Physical Chemistry Letters. 8(1):264278. doi:10.1021/acs.jpclett.6b01996

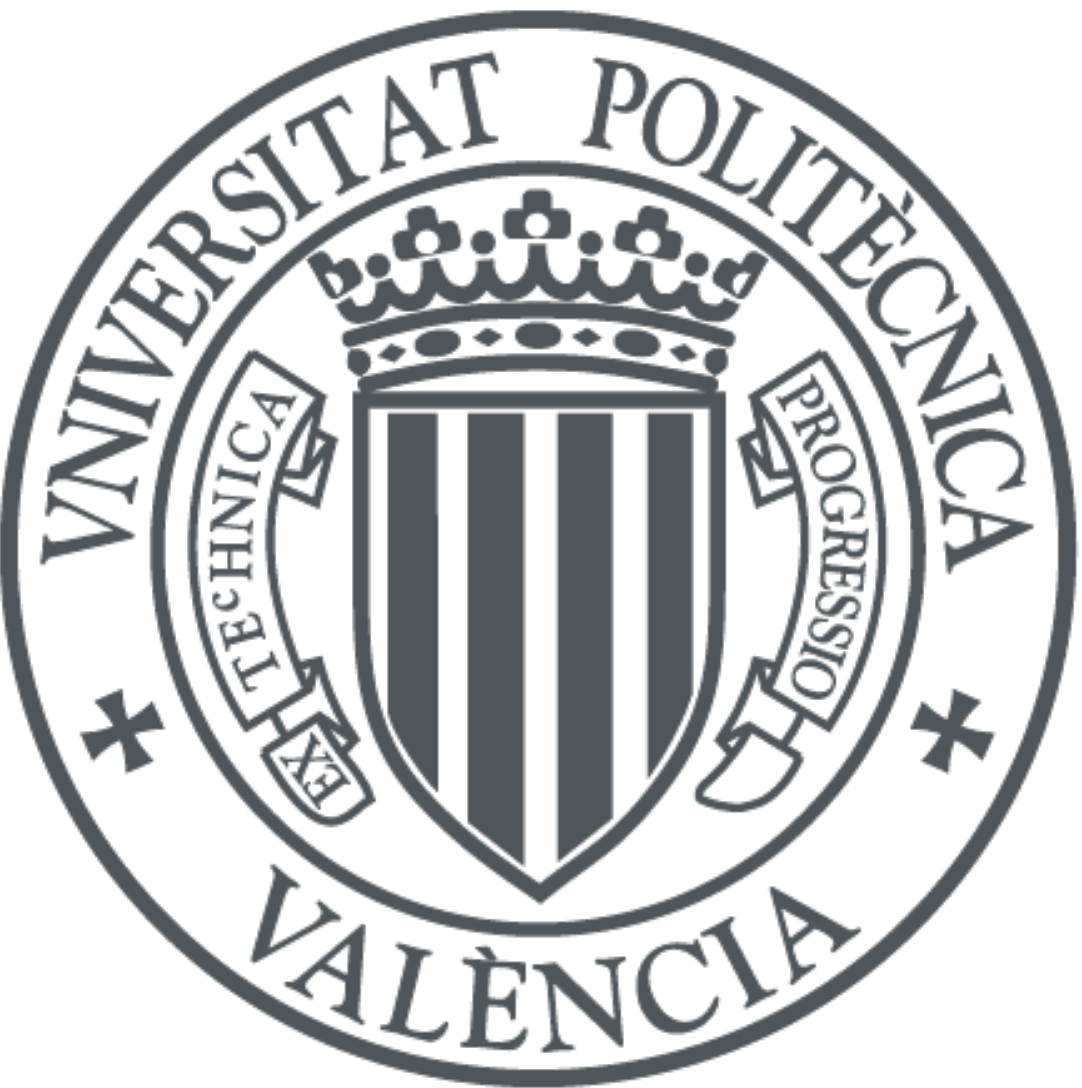

The final publication is available at

https://doi.org/10.1021/acs.jpclett.6b01996

Copyright American Chemical Society

Additional Information 


\title{
Graphenes as Metal-Free Catalysts with Engineered Active Sites
}

\author{
Ana Primo, ${ }^{a}$ Vasile Parvulescu ${ }^{b^{*}}$ and Hermenegildo Garcia ${ }^{\mathrm{a}}$ \\ a Instituto de Tecnología Química CSIC-UPV, Universitat Politecnica de \\ Valencia, Av. de los Naranjos, s/n, 46022 Valencia, Spain \\ ${ }^{b}$ Department of Organic Chemistry, Biochemistry and Catalysis, Faculty of \\ Chemistry, University of Bucharest, Bdul Regina Elisabeta 4-12, Bucharest 030016, \\ Romania
}

\begin{abstract}
This Perspective article highlights how recent discoveries on the activity of defective graphene to promote different organic reactions as metal-free catalysts has led to propose certain substructures present on these defective graphenes as active sites. The sustainability of using as catalysts graphenes obtained from biomass, together with the possibility to generate active sites by introducing defects on the sheet are the two main characteristics that are triggering research in this area. Emphasis is made in the need of gaining understanding on the nature of the active sites and how this understanding requires the combination of conventional kinetic experiments as well as advanced characterization tools. The relationship between catalysis by graphene and organocatalysis has also been remarked.
\end{abstract}




\section{Interest of metal-free catalysis}

A large percentage estimated in about $80 \%$ of chemical industrial processes are catalytic reactions. ${ }^{1}$ Catalysis is nowadays dominated by the use of transition metals, some of them considered as noble and critical metals of which there are limited available resources. $^{2}$ To avoid dependency of strategic sectors on precious and critical metals there is a large incentive in finding alternatives to some of the current metal catalysts. ${ }^{2,3}$ Scheme 1 summarizes some of the research lines in heterogeneous catalysis that have been developed to reduce the dependency on noble and critical metals

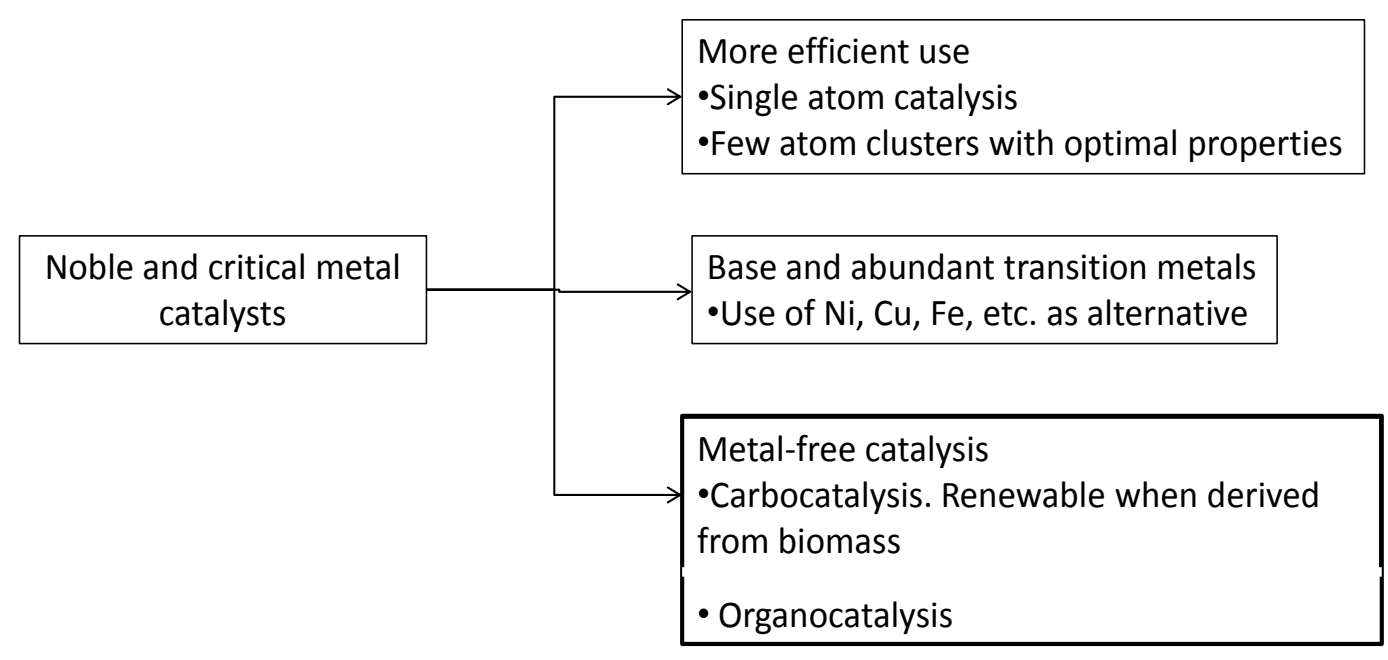

Scheme 1. Possible alternatives to the use of precious and critical metals.

One obvious possibility is to make a much better use of the limited amount of these metals by developing more efficient catalysts that should allow diminishing the metal consumption by improving the activity per site. The present metal catalysts can be improved by decreasing the size of the particles, even reaching the state of single atomsite catalyst and by increasing their stability and reactivation protocols. ${ }^{4,5}$ It should be commented that current data have determined that the number of metal atoms in a cluster to have optimal activity varies depending on the reaction and can be from 5 to 10 atoms. $^{4,6,7}$ In any case, this strategy would require control of metal nanoparticles 
(MNPs) and clusters with atomic precision that could eventually lead to optimal catalysts with clusters of the exact number of atoms required in the reaction mechanism. ${ }^{6,8}$ By reaching this goal a substantial diminution in the amount of precious metal can be achieved. However, this strategy should overcome satisfactorily the general problems of how to produce them in sufficiently large quantities and how to stabilize these highly reactive small clusters avoiding their agglomeration under reaction conditions, without at the same time decreasing their activity. In this regard, the use of MNPs, instead of metal clusters, can be considered in a certain way as the current solution for this dilemma, finding a balance between the minimum number of metal atoms and stability.

A radically different possibility consists in developing suitable alternative to critical metals, such as replacing them by abundant base transition metals or even the use of metal-free catalysts. One of the most fruitful approaches so far for metal-free catalysis has been the use of carbon-based materials. ${ }^{9,}{ }^{10}$ For the sake of sustainability and affordability, a favorable situation will be to prepare carbon-based catalysts derived from biomass. Particularly interesting would be the use of biomass wastes as catalyst precursors, since this approach would not compete with the production of food, but rather will complement it by valorization of biomass residues. ${ }^{11,12}$

Carbocatalysis was known since long time, using initially active carbons. ${ }^{13}$ Reactions like NOx decomposition or oxidation of pollutants have been reported to be catalyzed by active carbons in the $90 \mathrm{~s} .{ }^{14}$ Active carbons have also been used in as oxidation catalysts in desulfuration of air and fuels, ${ }^{15}$ in the combustion of ammonia and $\mathrm{H}_{2} \mathrm{O}_{2}$ decomposition, ${ }^{16}$ oxidative dehydrogenation of ethylbenzene, ${ }^{17}$ among many other reactions. However, since the structure of active carbons is ill-defined and there is a large complexity in the distribution and location of various functional groups, there was 
never an intense research front comparable to those in metal catalysis trying to exploit in a systematic way the potential of carbons. The main reason was the lack of understanding on the nature of the active sites, the difficulty in their modeling and the limited control on how to increase their number and tune their properties.

\section{Graphene as carbocatalyst}

This area of carbocatalysis has received, however, a renewed attention since novel carbon allotropes, and particularly carbon materials rather than molecular carbon forms, have been discovered. ${ }^{18}$ The main advantage of carbon allotropes respect to active carbons is the well-defined structure of the ideal materials and the better and safer characterization of these materials, due to their much simpler and regular structure. Scheme 2 illustrates some of the carbon allotropes that have been used as carbocatalysts.

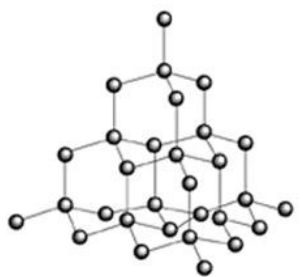

Diamond nanoparticles

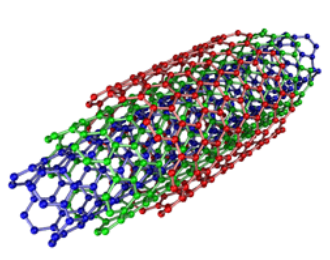

Multi wall carbon nanotubes

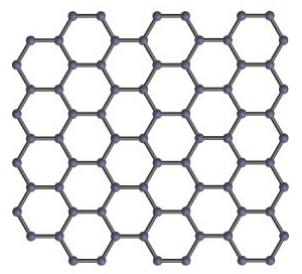

Graphene

Scheme 2. Three different carbon allotropes that have been used as metal-free catalysts.

Diamond nanoparticles are metal-free catalysts, for instance, for the oxidative dehydrogenation of alkanes to alkenes and they are quite robust. ${ }^{19}$ However, $\mathrm{sp}^{3}$ carbons cannot establish specific interactions with substrates and reagents other than strong 
irreversible covalent bonds or very weak van der Waals forces. In comparison, the graphene walls of carbon nanotubes (CNTs) are suitable to adsorb and interact with substrates by relatively strong $\pi$ interactions ${ }^{20}$ and the presence of defects on the ideal graphene structure can introduce some active sites. Thus, CNTs can be active to promote different reactions including the oxidative dehydrogenation of hydrocarbons, ${ }^{21}$ but also reduction of C-C multiple bonds by $\mathrm{NaBH}_{4}$ or hydrazine under aerobic conditions. $^{22,23}$

Carbocatalysis has, however, received a considerable impetus since the discovery of graphene $(\mathrm{G})$. The main reason is the large availability of $\mathrm{G}$ in sufficient quantities by various methods and the ease in which Gs can be modified and derivatized. Although, carbocatalysis using $\mathrm{G}$ is a continuation in many respects of that initiated by CNTs, the availability and versatility of Gs offer new opportunities and additional possibilities. Thus, although certain multi-wall CNTs are produced industrially in multiton scale, ${ }^{24}$ mainly for their use as battery additives, ${ }^{25}$ the large proportion of metallic catalysts required in the synthesis of CNTs is still a limitation, particularly to develop metal-free catalysis. Complete removal of trace quantities of metals from CNTs is problematic, particularly considering the possibility that these metals can become incorporated inside the nanotube. Also, reliable preparation procedures for the synthesis of single wall or CNTs with a controlled number of walls as well as with the presence of dopants are still required.

In contrast, there exist different preparation procedures of doped Gs. ${ }^{26,} 27$ Basically there are two alternative strategies, either introducing the dopant during the synthesis of $\mathrm{G}$ or using graphene oxide (GO) as precursor and grafting of the dopant element, while $\mathrm{GO}$ is being reduced to a defective type of $\mathrm{G}$, known as reduced 
graphene oxide (rGO). For all these reasons, there is much current interest in exploring the possibilities that Gs can offer as metal-free heterogeneous catalysts.

There exist in the literature several reviews covering exhaustively the type of reactions that have been reported using $G$ as carbocatalysts. ${ }^{18,}{ }^{26-29}$ In the present Perspective article and for the sake of space limitation, we will focus to the nature of the active sites that can be encountered in Gs and some of the types of chemical reactions that can promote. Active sites are always associated to defects in the structure of ideal G. Scheme 3 shows a pictorial illustration of some of the sites that have been proposed to be active in catalysis.

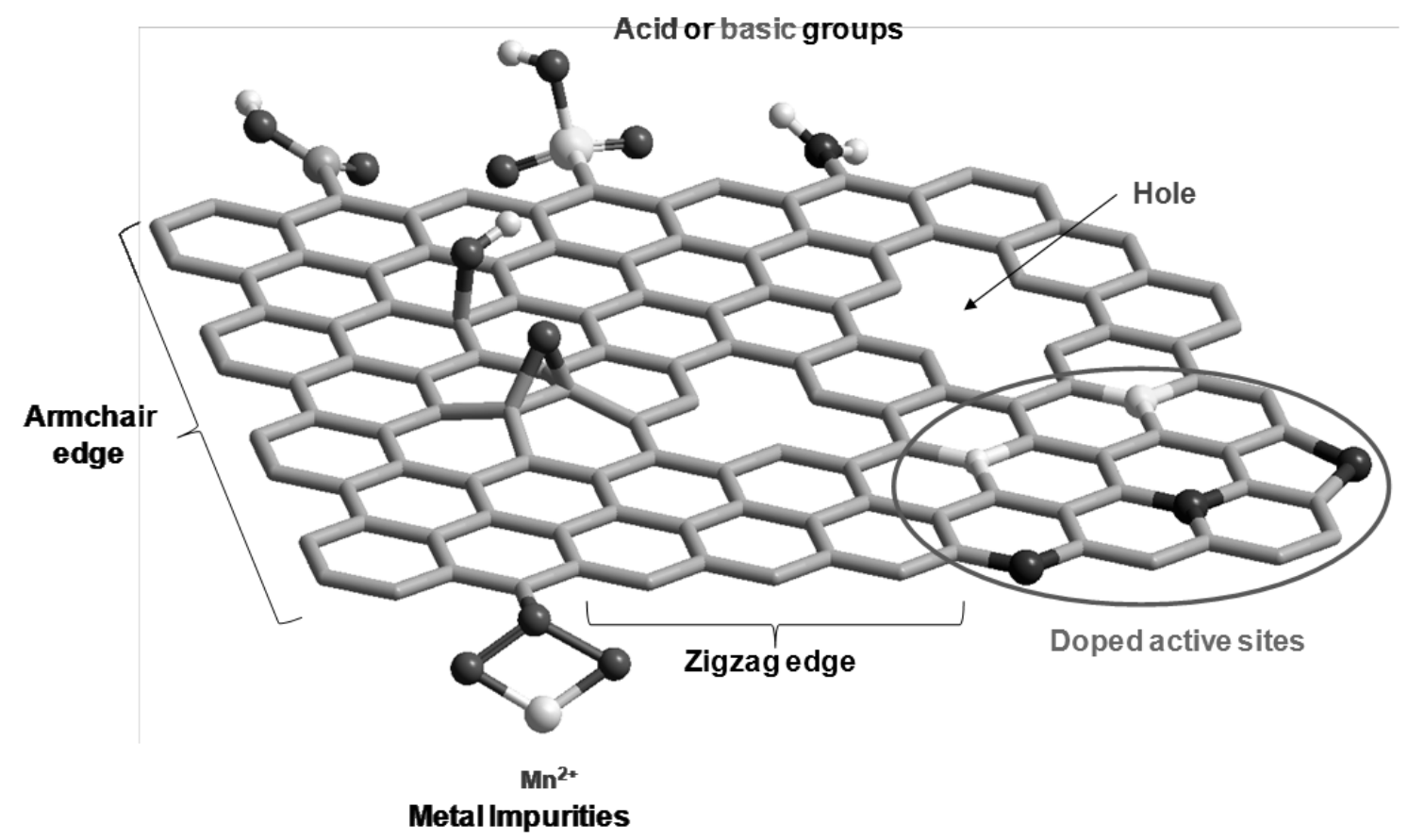

Scheme 3. Pictorial illustration of the types of defects of the ideal G structure that can become active sites in catalysis.

Most of the content of this Perspective article is derived from our own work, but references to other contributions will also be made. During this article, the close 
relationship between carbocatalysis and organocatalysis with regard to the possibility to have common types of active sites will be commented.

\section{Frustrated Lewis acid-base pairs as hydrogenation active sites}

G obtained by pyrolysis of alginate is able to promote the selective gas-phase hydrogenation of acetylene in the presence of a large excess of ethylene. ${ }^{30}$ Alginate is a natural polysaccharide present in a large percentage in brown algae and that is able to form defectless films of nanometric dimensions. ${ }^{31}$ The tendency of carbohydrates to form graphitic carbons under physical or chemical treatments was well-known since long ago, but the recent discovery is that these carbon residues resulting from the pyrolysis of filmogenic carbohydrates, like alginate, chitosan and carrageenans, can undergo easy exfoliation upon sonication giving rise to defective $G$ suspensions in a very high yield. ${ }^{32}$ The process is indicated in Scheme 4. This behavior of the alginate pyrolysis residue contrasts with highly crystalline graphite that essentially does not undergo exfoliation to deliver $\mathrm{G}$ under the same or harsher conditions. In addition, the $\mathrm{G}$ sheets obtained by alginate pyrolysis still contain a residual percentage of $\mathrm{O}$ or other heteroatoms present on the polysaccharide such as $\mathrm{N}$ or $\mathrm{S}$. In addition evolution of $\mathrm{CO}_{2}$ in the process generates carbon vacancies and holes (Scheme 3). The available characterization data of these $\mathrm{G}$ materials obtained from biomass indicate the presence of defects that are suitable to act as catalytic centers promoting different reactions, such as hydrogenation. In many respects, the $\mathrm{G}$ obtained by pyrolysis of alginate has many similarities with rGO formed by reduction of GO. 


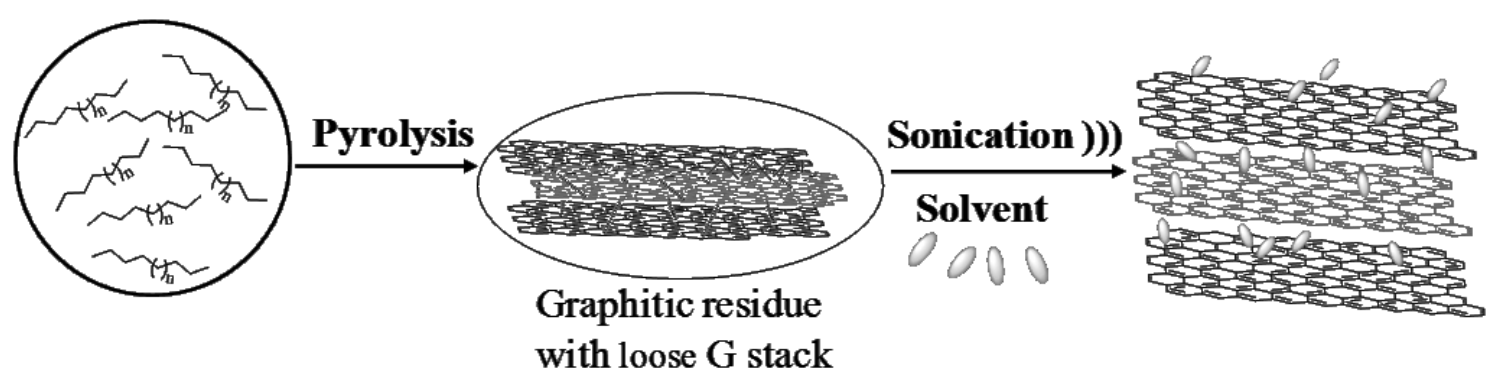

Scheme 4. Pyrolysis of natural filmogenic polysaccharides to render defective $G$ suspensions (taken from ref. ${ }^{27}$ ).

Selective acetylene hydrogenation has interest in refineries as a way to purify cracking streams in which the presence of few percents of acetylene is highly detrimental due to its tendency to polymerize and form gums and residues. Considering the availability of hydrogen in refineries and the difficulty to separate acetylene from ethylene due to their similar boiling points and other physical properties, one possibility would be to convert acetylene into ethylene that has the additional advantage of increase the overall ethylene yield. Scheme 5 illustrates the process. But, hydrogenation of acetylene to ethylene in the presence of a large excess of the latter requires of a highly selective catalyst, like $\mathrm{G}$ derived from alginate.

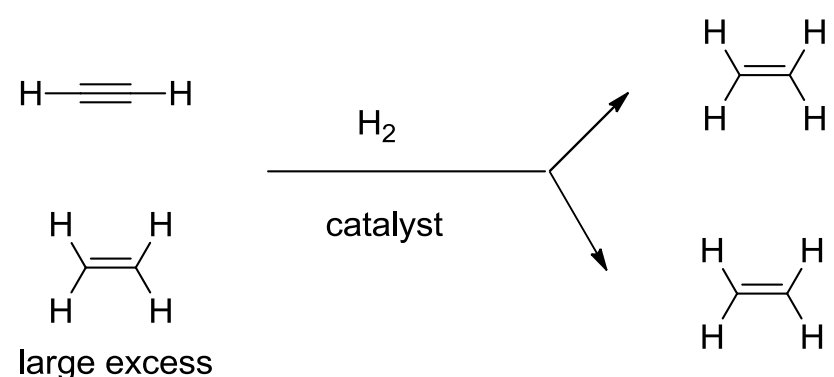

Scheme 5. Selective hydrogenation of acetylene in the presence of ethylene.

Other related G types tested were not efficient, particularly doped Gs that are not stable under the reaction conditions and undergo hydrogenative removal of the dopant element in a significant extent. ${ }^{30}$ For instance, in the case of N-doped G, obtained in this 
case by pyrolysis of chitosan and subsequent exfoliation, formation of $\mathrm{NH}_{3}$ was detected under the conditions of selective acetylene hydrogenation and XPS reveals that this partial dopant removal is also the case of $\mathrm{P}$ and S-doped Gs. ${ }^{30}$

While selective acetylene hydrogenation is a gas-solid process, defective $\mathrm{G}$ from alginate exhibited also catalytic activity for hydrogenation of alkenes, such as cyclohexene and styrenes, in the liquid phase. ${ }^{30}$ Latter the catalytic activity rGO and $\mathrm{G}$ from alginate has been expanded to the hydrogenation of nitro groups, although the process is not selective and simultaneous hydrogenation of $\mathrm{NO}_{2}$ and $\mathrm{C}=\mathrm{C}$ occur when both functional groups are present in the molecule. ${ }^{33}$

Due to the importance of catalytic hydrogenation in industry as well as in organic synthesis, understanding of the nature of the active sites is crucial to develop more efficient carbocatalysts, particularly considering that comparison of the activity of Gs with that of Pd or other metals has shown that Gs are significantly less active. ${ }^{30}$ Organocatalysis has shown that organic molecules containing simultaneously a Lewis acid and a Lewis base center at a short distance, but without reacting each other due to steric hindrance, are able to promote metal-free hydrogenations. ${ }^{34}$ Scheme 6 presents one of these molecular hydrogenation catalysts. The type of active centers present in these molecules has been denoted as "frustrated Lewis acid-base pairs" and theoretical calculations as well as experimental evidence has shown that they can activate molecular hydrogen. ${ }^{34}$ 

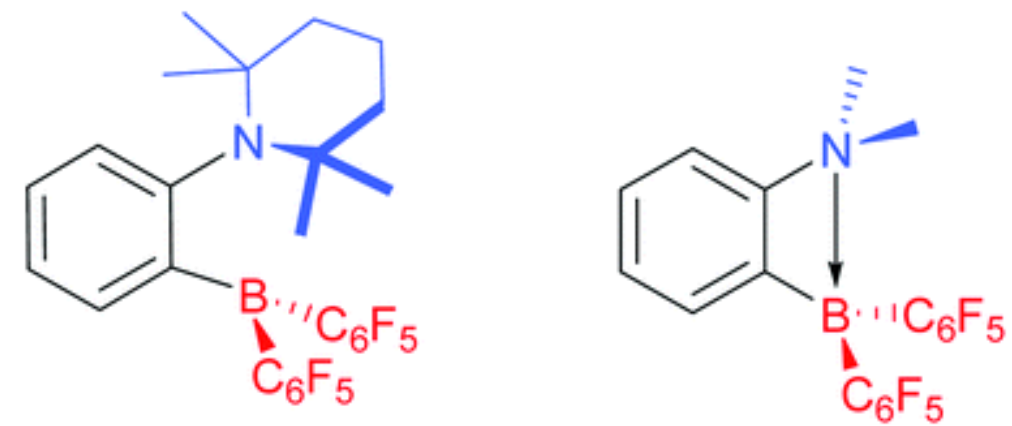

Scheme 6. Illustration of how steric encumbrance around the basic part of the molecule (blue) can impede its interaction with the Lewis acid moiety (red) of the left molecule, rendering a frustrated Lewis pair that is able to activate molecular $\mathrm{H}_{2}$ in contrast to the right analogue without steric hindrance (taken with permission from ref. $\left.{ }^{35}\right)$

It has been proposed that similar types of frustrated Lewis pairs could be present on $\mathrm{G}$, activating $\mathrm{H}_{2}$ molecule. Titrations by thermoprogrammed desorption of ammonia and $\mathrm{CO}_{2}$ as base and acid, respectively, has established the presence of both types of centers on defective G. At the same time, the reversible influence on the activity of $G$ for selective acetylene hydrogenation of the presence of $\mathrm{NH}_{3}$ or $\mathrm{CO}_{2}$ in the acetylene/ethylene flow also supports that acidity and basicity is important in the catalytic activity.

Theoretical calculations by $\mathrm{Su}$ and $\mathrm{L}$ have shown that $\mathrm{H}_{2}$ molecules within the interlayer space of two $\mathrm{G}$ layers one can be activated by graphitic $\mathrm{B}$ and $\mathrm{N}$ atoms at the appropriate distance (Figure 1). ${ }^{36}$ Although diffusion of $\mathrm{H}_{2}$ accessing the interlayer position to become activated can be problematic, these calculations prove that $\mathrm{H}_{2}$ activation can be possible using the frustrated Lewis pair concept provided that geometric requirements are met. One concern for this mechanism of $\mathrm{H}_{2}$ activation by $\mathrm{G}$ is the lack of control in the distribution of acid and basic sites. Thus, random 
distribution implies that only the minor fraction of the total acid and basic sites that meet the distance requirement are active in the reaction and, therefore, optimization of the catalytic activity of $\mathrm{G}$ materials for hydrogenation would require the development of advanced synthetic methods to gain a precise control on the distribution of sites on G.

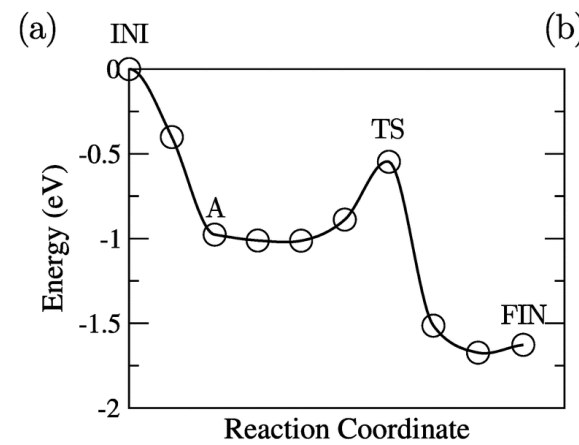

(b) INI (side view) INI (top view)
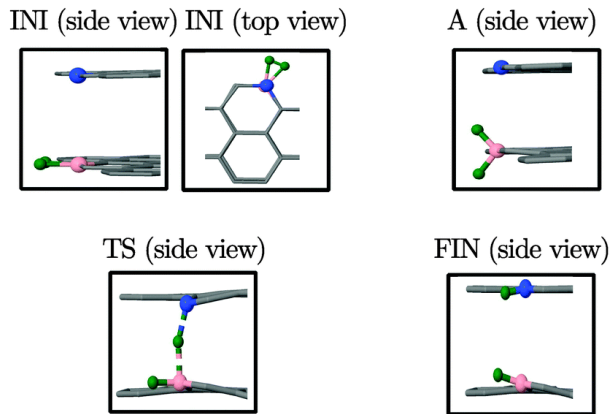

Figure 1. Energy profile of $\mathrm{H}_{2}$ activation and relevant states for the proposed mechanism for $\mathrm{H}_{2}$ activation by frustrated Lewis pairs constituted by graphitic $\mathrm{N}$ and $\mathrm{B}$ atoms in bilayer $\mathrm{N}, \mathrm{B}$-codoped $\mathrm{G}$ (taken with permission from ref. ${ }^{36}$ ).

However, to illustrate the current knowledge, it should be said that, besides frustrated Lewis pairs, other possible defects on $\mathrm{G}$ have also been proposed as sites for $\mathrm{H}_{2}$ activation. Thus, theoretical studies have also concluded that the edges of $\mathrm{G}$, particularly the zig-zag having or not heteroatoms, can activate $\mathrm{H}_{2} \cdot{ }^{37,38}$ If borders of $\mathrm{G}$ would have the ability to activate $\mathrm{H}_{2}$, then, the smallest the $\mathrm{G}$ lateral size and larger the periphery of the sheets, the larger should be the catalytic activity, Catalytic data of G nanosheets would be valuable to address the possible $\mathrm{H}_{2}$ activation by the borders. Also carbon vacancies have been suggested as $\mathrm{H}_{2}$ activation sites, according to DFT calculations ${ }^{39,40}$ and, also in this case, experimental evidence supporting this proposal could be gained by comparing the activity of various $\mathrm{G}$ samples having different density of holes on the sheet, something that seems doable considering that density of holes can be quantified by TEM. 


\section{Carbon vacancies as initiators of aerobic oxidations.}

Oxidation of hydrocarbons and other organic molecules by oxygen is one reaction type that has considerable importance in industry. ${ }^{1}$ Among the industrial aerobic oxidations now in use, oxidation of benzylic positions of alkylaromatics, oxygenation of cyclic and acyclic alkanes and epoxidation of alkenes are well-known examples.

There are several possible mechanisms for activation of $\mathrm{O}_{2}$, including electron transfer with the formation of $\mathrm{O}_{2}{ }^{--}$superoxide and other reactive oxygen species derived therefrom, generation of singlet oxygen and generation of carbon centered radicals able to react directly with $\mathrm{O}_{2}{ }^{41}$ Aerobic oxidations involving carbon centered radicals are generally denoted as "autooxidations" and consist in a chain mechanism in which carbon centered radicals react without activation barrier with ground state, triplet oxygen to give a peroxyl radical that upon hydrogen abstraction from the substrates initiates another cycle. Scheme 7 summarizes the main steps in this general reaction with oxygen.

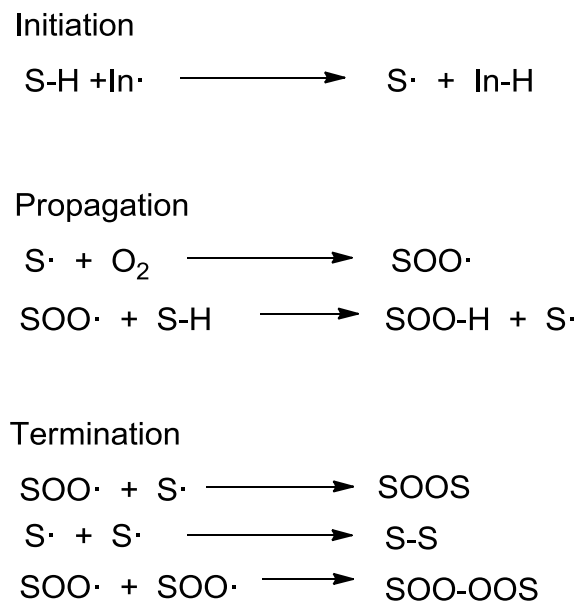


Scheme 7. The three steps of the chain mechanism of aerobic oxidations. Note that the propagation steps controlling the reaction rate do not require any catalyst. S-H: Substrate; In $\because$ initiator.

As indicated in Scheme 7, this mechanism requires an initiation step consisting in the generation of the first carbon centered radical that can result in the formation of tens to thousands of product molecules depending on the chain length, i.e., the average number of turnovers in the propagation steps. Since the propagation steps do not require any catalyst, due to the low activation barriers involved, autooxidations can be carried out by providing an initiator that is responsible of the generation of the initial carbon radicals. Thus, the role of Gs or other initiators such as classical azo bis(isobutyronitrile) is the generation of the primary radicals (In· in Scheme 7) that by hydrogen abstraction form the carbon radical of the substrate.

Although Gs, GO and related materials can promote aerobic benzylic oxidations and autooxidation of other substrates, ${ }^{42-44}$ it is arguable that these reactions should be considered as "catalytic" as they are generally described in the literature. In any case, there is abundant information showing that Gs can trigger the generation of carbon radicals that in the presence of $\mathrm{O}_{2}$ result in the oxidation of a wide range of substrates, such as benzylic hydrocarbons, ${ }^{45}$ alcohols, ${ }^{43,46,47}$ and amines, ${ }^{9}$ (cyclo)alkanes, alkenes, thiols. ${ }^{48}$ The reaction mechanism of autooxidations can be proved by adding radical quenchers that should stop the reaction, while they are still present. Typical quenchers are phenols, but also TEMPO and stable $\mathrm{O}$ centered radicals. In addition, it is also quite common to observe an induction period in the time-conversion plot that is related with the initiation step and the generation of the first radicals. 
It has been proposed that carbon vacancies and dangling bonds associated to defects are the most likely sites that can act as initiators in these aerobic oxidations. ${ }^{9,45}$ This is reasonable considering that carbons on $\mathrm{G}$ with incomplete valence should have some radical character that can act as initiators. In general, it seems that doping increases the activity of Gs as promoters of aerobic oxidations, therefore, it can be inferred that the dopant elements should also play a role in the activation of radical generation, although more insights on the reasons are needed. In the case of GO, where there is a large percentage of $\mathrm{O}$, about $50 \%$, theoretical calculations for benzyl alcohol oxidation to aromatic aldehydes have led to propose that there is a hydrogen transfer from alcohol substrate to epoxide groups on $\mathrm{F}$ that undergo ring opening, followed by dehydration. ${ }^{49}$ This change in the nature of the active site depending on the aerobic oxidation and the type of $\mathrm{G}$ is also of large potential interest that can be exploited for controlling the selectivity of the process.

Aerobic oxidations initiated by Gs can gain importance in the context of novel process for biomass conversion. Cellulose is the main component of biomass and it can be hydrolyzed using GO to glucose. ${ }^{50}$ Subsequently, glucose can be submitted to wet oxidation to afford succinic acid with a selectivity over $60 \%$ using Gs to drive the selectivity to this wanted dicarboxylic acid. ${ }^{51}$ Scheme 8 summarizes the steps for preparation of succinic acid from cellulose. Succinic acid from glucose using $\mathrm{O}_{2}$ as reagent can be a key process in next generation polyesters based on biomass.

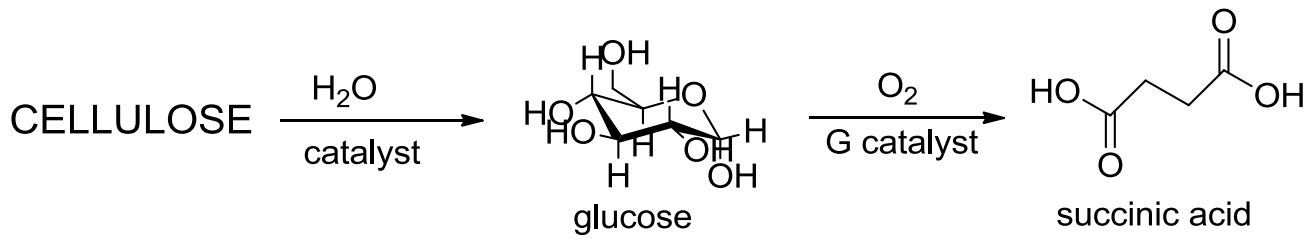


Scheme 8. Preparation of succinic acid from cellulose in two steps, the last one being the wet oxidation catalyzed by $\mathrm{G}$.

Besides cellulose, lignin is the second major constituent in biomass. Depolymerization of lignin is one of the main problems for its use as source of chemicals. Using $\alpha$-guaiacylglyceryl- $\beta$-guaiacol as model of some of the strong ether bonds present in lignin, it has been found that this probe molecule can be fragmented by $\mathrm{O}_{2}$ using rGO as catalyst (Scheme 9$){ }^{52}$

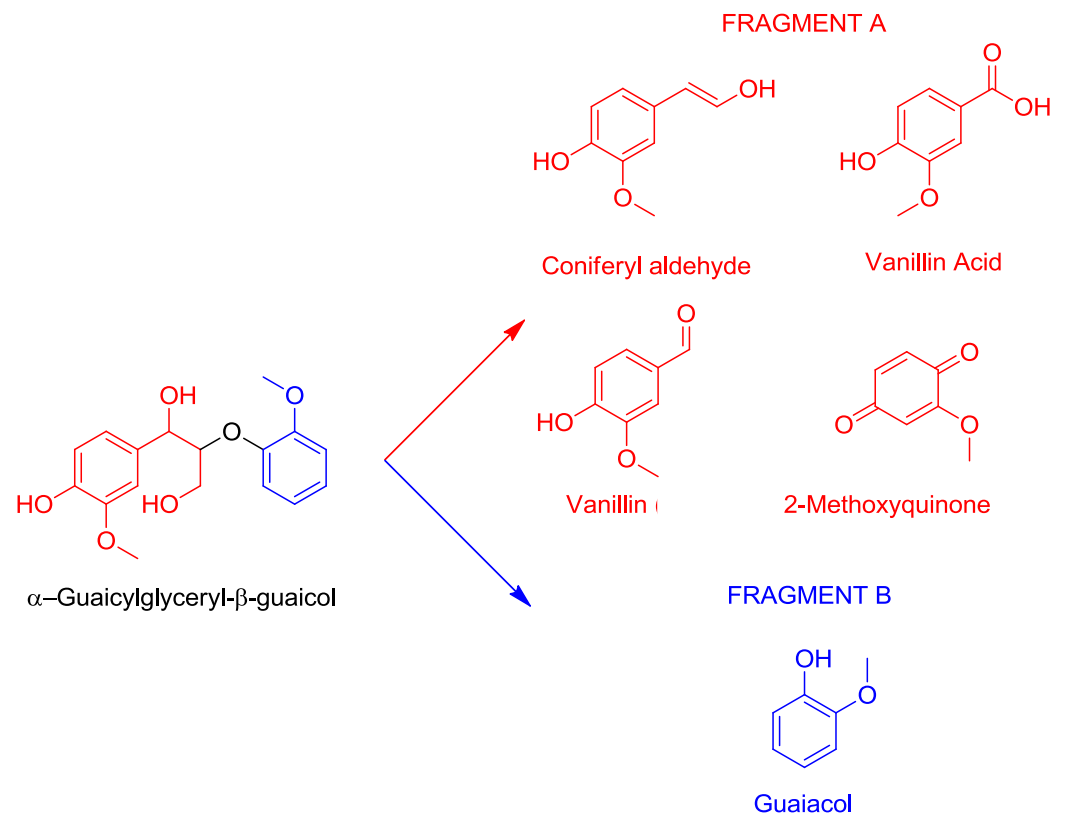

Scheme 9. Oxidative cleavage of an ether bond promoted by rGO in a molecule considered as a model of this type of bonds in lignin. ${ }^{52}$

\section{Quinone/hydroquinone like substructures as oxidation sites}

The previous section describes promotion of aerobic oxidation through a radical chain mechanism, but there are many other oxidation reactions using a wide range of oxidants and occurring through different reaction mechanisms. Depending on the 
reaction, the nature of the active sites could change, as already commented in the case of aerobic oxidation of benzyl alcohols in the presence of $\mathrm{GO},{ }^{49}$ and this could result in a change in the product selectivity. A clear example of this change in the nature of the active sites depending on the oxidizing reagent and in the product distribution has been observed in the case of primary amines using $\mathrm{NaClO}$ as oxidant and $\mathrm{GO}$ as catalyst. ${ }^{53} \mathrm{In}$ this case, the main oxidation product depends on the substrate, but for long chain aliphatic amines the major products are the corresponding nitriles (Scheme 10). ${ }^{53}$ FT-IR spectroscopy supports that in this case, carboxylic acid groups forming peroxyacids or peroxylactones are the active sites. A clear analogy with carbocatalysis by $\mathrm{G}$ and organic chemistry in which peracids are well-known oxidizing reagents ${ }^{54}$ is clearly established.

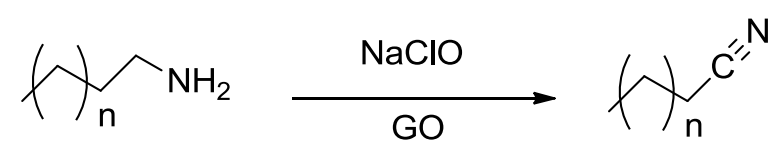

$\mathrm{n} ; 9,11,13$

Scheme 10. Oxidation of primary long-chain aliphatic amines to the corresponding aliphatic nitriles with almost complete selectivity.

Another oxidation process that has wide application for waste water treatment when the amount of organic matter is not very low or high ranging from 1 to $20 \mathrm{ppms}$ is the Fenton reaction using hydrogen peroxide to form hydroxyl radicals $(\cdot \mathrm{OH})$ in water. ${ }^{55}$ Typically the Fenton reaction is carried out using stoichiometric amounts of $\mathrm{Fe}^{2+}$ salts to reduce $\mathrm{H}_{2} \mathrm{O}_{2}$ according to eq. 1. According to this eq. 1, formation of $\cdot \mathrm{OH}$ radicals from $\mathrm{H}_{2} \mathrm{O}_{2}$ is a one-electron reduction and, besides, $\mathrm{Fe}$, it has also been reported for other transition metal salts, such as $\mathrm{Cu}$ and $\mathrm{Mn} .^{55}$ 


$$
\mathrm{Fe}^{2+}+\mathrm{H}_{2} \mathrm{O}_{2} \longrightarrow \mathrm{Fe}^{3+}+\cdot \mathrm{OH}+\mathrm{OH}^{-} \quad \text { eq. } 1
$$

There is a large practical incentive in developing efficient Fenton catalysts to overcome the need of large amounts of metals of stoichiometric reactions. Supported Fe oxides and metal NPs have been reported as catalysts for this reaction, ${ }^{55,56}$ although in most of the studies a large excess of $\mathrm{H}_{2} \mathrm{O}_{2}$ over substrate that can be of 1000 equivalents or even larger has been employed.

Since active carbons are widely used in water purification, there is a very large number of publications reporting the use of active carbons of various types for the socalled advanced oxidation processes, ${ }^{57}$ where the oxidant can be either oxygen (catalytic wet oxidation), ${ }^{58-60}$ hydrogen peroxide (catalytic peroxidation) ${ }^{61,62}$ or ozone ${ }^{63}$. In particular, it has been established that metal-free carbons of basic nature are excellent catalysts for these reactions, including nitrogen-doped CNTs ${ }^{64}$ and graphene derived materials.

In this context, using phenol as a model molecule for reluctant, nonbiodegradable organic pollutant it has been found that defective $\mathrm{G}$ obtained by pyrolysis of alginate is a convenient catalyst that is able to promote Fenton degradation using a minimal excess of 5.5 equivalents of $\mathrm{H}_{2} \mathrm{O}_{2} \cdot{ }^{65}$ Compared to other $\mathrm{G}$ catalysts, defective $\mathrm{G}$ from alginate was found more efficient than rGO and doped Gs. Noteworthy is the lack of catalytic activity of GO to promote this reaction. Figure 2 depicts the temporal profiles for phenol degradation and $\mathrm{H}_{2} \mathrm{O}_{2}$ decomposition in the presence of various $\mathrm{G}$ catalysts. 


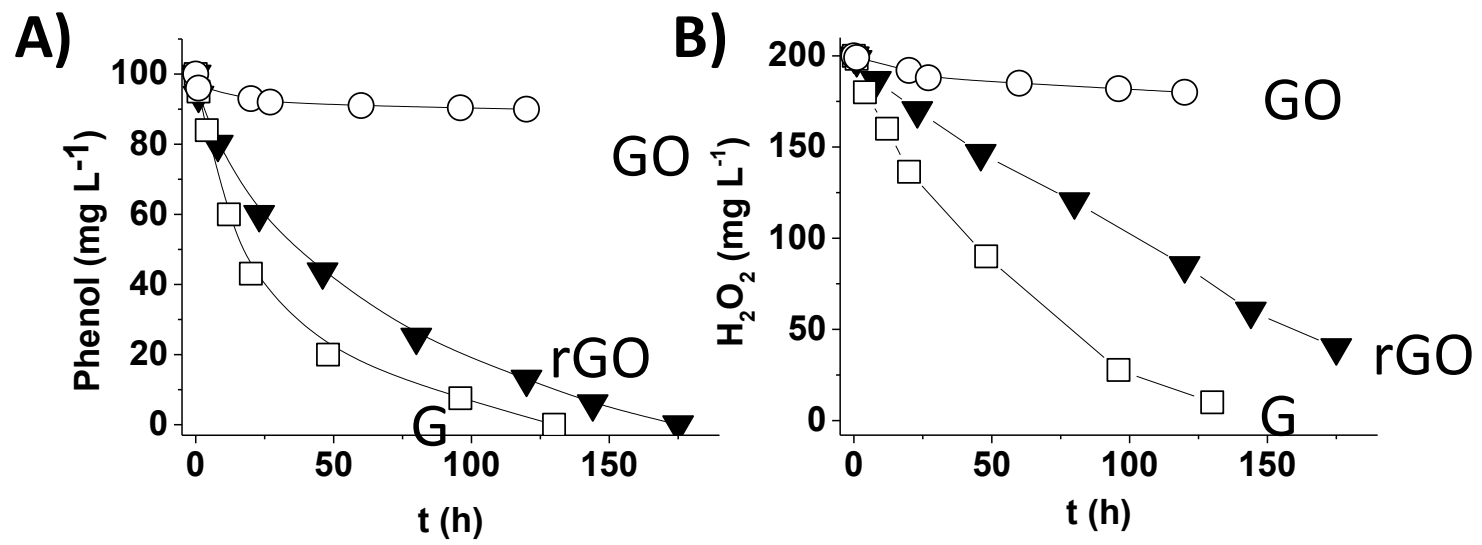

Figure 2. Temporal profiles of phenol degradation (A) and consumed $\mathrm{H}_{2} \mathrm{O}_{2}$ (B) using three G-based catalysts. $G$ corresponds to defective $G$ obtained from alginate pyrolysis at $900{ }^{\circ} \mathrm{C}$. Reaction conditions: Catalyst $\left(200 \mathrm{mg} \mathrm{L}^{-1}\right)$, phenol $\left(100 \mathrm{mg} \mathrm{L}^{-1}\right.$, $1.06 \mathrm{mM}), \mathrm{H}_{2} \mathrm{O}_{2}\left(200 \mathrm{mg} \mathrm{L}^{-1}, 5.88 \mathrm{mM}\right), \mathrm{pH} \mathrm{3,} 20^{\circ} \mathrm{C}$. (Taken from ref. ${ }^{65}$ ).

Noteworthy is that for defective $G$ the activation energy for phenol degradation coincides with that of $\mathrm{H}_{2} \mathrm{O}_{2}$ decomposition as it should be expected if highly aggressive - $\mathrm{OH}$ radicals were the only species formed from $\mathrm{H}_{2} \mathrm{O}_{2} \cdot{ }^{65}$ Moreover, the process can be also activated by natural sunlight, probably reflecting the photocatalytic activity of these defective G. ${ }^{66}$

Mechanistic studies using selective quenchers and, particularly unambiguous EPR detection of the adduct of $\cdot \mathrm{OH}$ trapping by phenyl N-tert-butyl nitrone, has provided firm support of the generation of $\cdot \mathrm{OH}$ radicals by defective $\mathrm{G} .{ }^{65}$ Calculations have suggested that the hydroquinone/quinone redox pair with similar redox potential as the $\mathrm{Fe}^{2+} / \mathrm{Fe}^{3+}$ pair could be the active sites. This proposal was independently supported by using differently substituted hydroquinones and quinones as organocatalysts and by detecting the generation of $\cdot \mathrm{OH}$ radicals. ${ }^{65}$ It should be noted that since $\mathrm{H}_{2} \mathrm{O}_{2}$ can act as reducing agent forming $\mathrm{O}_{2}\left(\mathrm{E}_{\mathrm{red}}{ }^{\mathrm{o}} 0.68 \mathrm{~V}\right)$ and oxidizing agent forming $\mathrm{H}_{2} \mathrm{O}\left(\mathrm{E}_{\text {red }}{ }^{\mathrm{o}} 1.78\right.$ $\mathrm{V})$, it is not relevant from the catalytic point of view to start from hydroquinones or 
quinones, provided that the redox potential of the hydroquinone/quinone pair is in between the range of potentials of the two redox processes of $\mathrm{H}_{2} \mathrm{O}_{2}$ (Scheme 11).

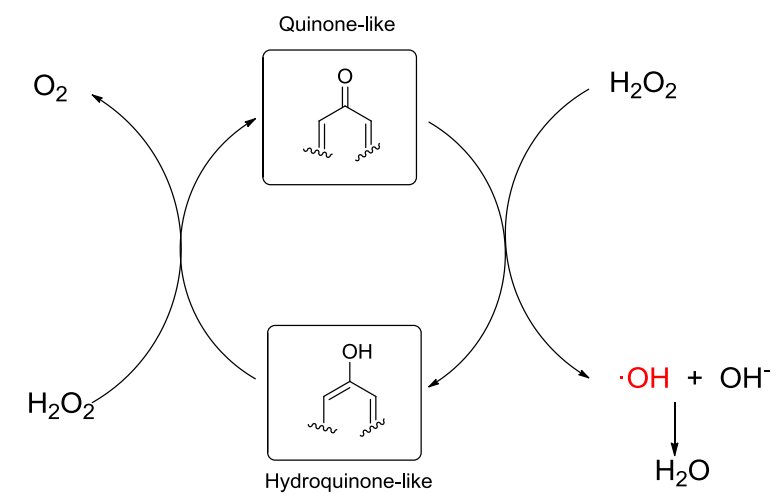

Scheme 11. $\mathrm{H}_{2} \mathrm{O}_{2}$ decomposition catalyzed by hydroquinone-quinone pair. Note that $\mathrm{H}_{2} \mathrm{O}_{2}$ can behave as oxidizing and reducing reagent.

Again, to support the nature of active sites on Gs, an analogy with the activity of organic molecules was established. ${ }^{65}$ Scheme 12 shows some thermodynamic data, $\Delta \mathrm{E}$, i.e. the difference in energy between the products and the reagents, showing that the process is moderately endoergonic by 1 or $2 \mathrm{eV}$ in a difference between terms of about $130 \mathrm{eV}$. Depending on the substituent, as in the case of condensed bis pyreno hydroquinone (Scheme $12 \mathrm{~b}$ ), the difference is just $1 \mathrm{eV}$. It should be commented that from the point of view of catalysis, the ideal situation is quasi neutral process $(\Delta \mathrm{E} \sim 0$ $\mathrm{eV}$ ) from the energy point of view, allowing turnover from hydroquinone to quinone and viceversa without irreversible oxidation or reduction. 
a)<smiles>[R]C1=CC(=O)C=C([R])C1=O</smiles>

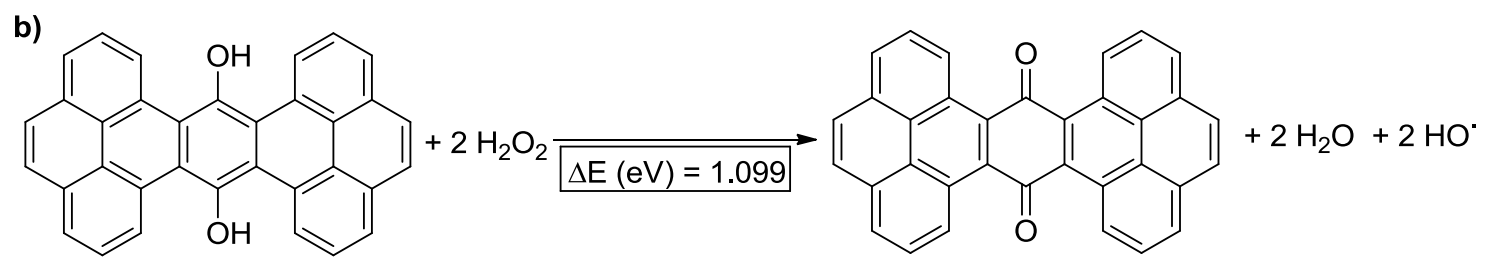

Scheme 12. Calculated energy data for the generation of $\cdot \mathrm{OH}$ radicals by reduction of $\mathrm{H}_{2} \mathrm{O}_{2}$ with hydroquinones (taken from ref. ${ }^{65}$ ).

In organic chemistry, it is well known that quinones, such as 2,3-dichloro-5,6dicyanoquinone (DDQ) are good oxidizing reagents ${ }^{67}$ and that hydroquinones are reducing agents. ${ }^{54}$ One main difference is, however, that these hydroquinone-quinone substructures in Gs are notable stable, since the $\mathrm{G}$ can be reused as catalyst and a turnover number higher than 15,000 for $\mathrm{H}_{2} \mathrm{O}_{2}$ decomposition has been measured considering that the number of centers present in $\mathrm{G}$ correspond to the fraction of $\mathrm{C}$ atoms bonded to $\mathrm{O}$ atoms as carbonyls.

This type of substructures in Gs in which a carbonyl group is attached to unsaturated sp2 carbons have also been generically denoted as oxygenated functional groups or more specifically carbonyl groups and have been proposed as active sites for different reactions. ${ }^{68}$

\section{Impurities as active sites.}

\subsection{Non-metallic impurities}


It has been determined that rGO can behave as acid catalyst for reactions such as aldehyde acetalization, ${ }^{69}$ epoxide ring opening ${ }^{70}$ and hydrolysis of biomass. ${ }^{71}$ It has been found, however, that this catalytic activity as acid correlates well with the percentage of $\mathrm{S}$ present in the $\mathrm{G}$ sample. ${ }^{69}$ Two possible functional groups, either hydrolyzable hydrogen sulfate $\left(\mathrm{C}-\mathrm{O}-\mathrm{SO}_{3} \mathrm{H}\right)$ or sulfonic acid $\left(\mathrm{C}-\mathrm{SO}_{3} \mathrm{H}\right)$ can be formed in the process (Scheme 13).

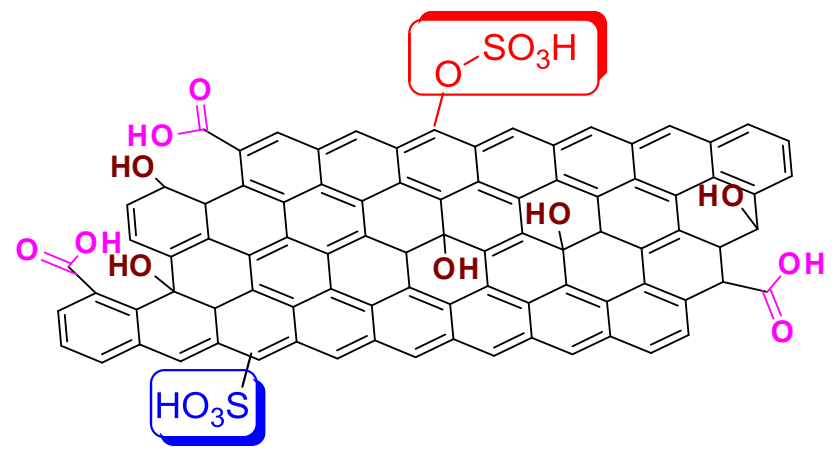

Scheme 13. Hydrogen sulfate (red) and sulfonic acid (blue) groups that can be formed during the process of rGO formation as consequence of the excess of sulfuric acid used in the Hummers oxidation of graphite.

In aldehyde acetalization ${ }^{69}$ and epoxide ring aperture, ${ }^{70}$ both processes carried out in methanol, it was found that product yields and sulfur content decreases upon reuse, indicating that most of the catalytic activity is due to the presence of hydrogen sulfate groups. Similar behavior of rGO as acid catalyst due to the presence of S species have been reported in the literature, ${ }^{73-75}$ for instance in the hydrolysis of cellulose or the isomerization-dehydration of glucose, although in this case the activity was attributed mainly to carboxylic acid groups. ${ }^{50}$

The activity of $\mathrm{G}$ anchored hydrogen sulfate groups is frequently much higher than equivalents amounts of $\mathrm{H}_{2} \mathrm{SO}_{4}$ or other acids in solution. ${ }^{50,70}$ For this reason sulfated $\mathrm{G}$ is a highly active catalyst for various biomass conversion processes, such as, 
for instance, degradative hydrolysis of starch to levulinic acid using a trifluorosulfonate $\mathrm{G}$ (Scheme 14). ${ }^{76}$

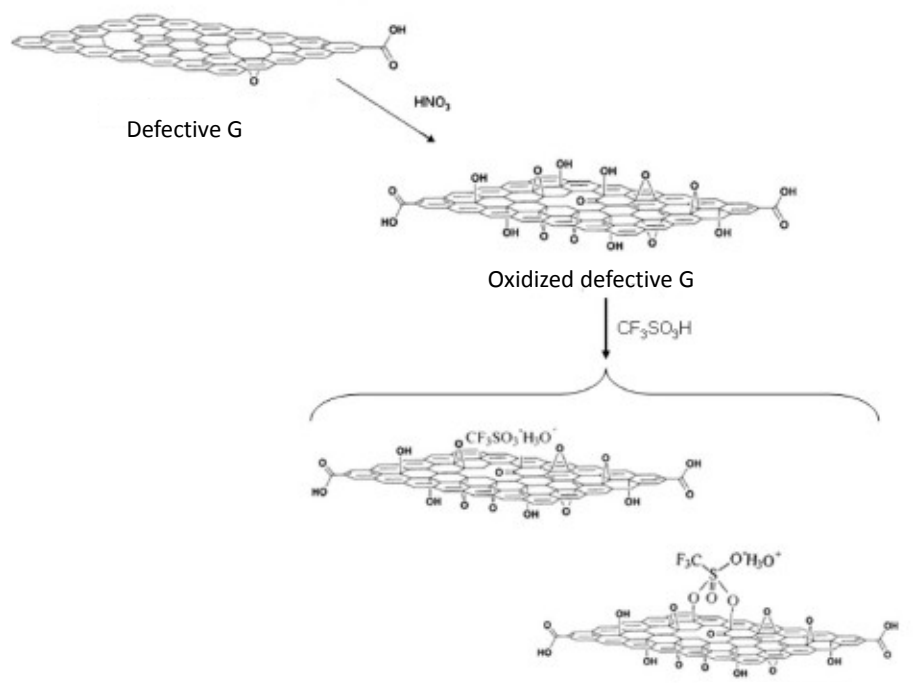

Scheme 14. Possible structures of trifluorosulfonated defective G. Note that defective $G$ was submitted to an oxidative pretreatment to increase the density of oxygenated groups. (Taken with permission from ref. ${ }^{76}$ ).

This synergy between the catalytic center and the morphology and properties of $\mathrm{G}$ is more general than just the case of acid sites as already commented ${ }^{77}$ and it can be taken advantageously in the case modified Gs having sulfonic groups covalently anchored through non-hydrolyzable bonds (Scheme 15).

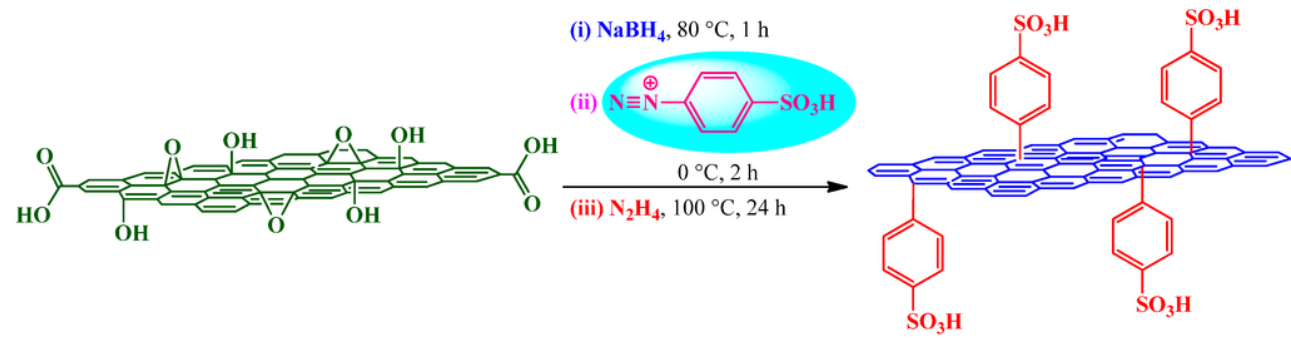

Scheme 15. Covalent anchoring of benzenesulfonic groups to $\mathrm{G}$ by reaction of diazonium salts with rGO (taken from ${ }^{73}$ ). 


\subsection{Metal impurities.}

Exhaustive analyses by Pumera and coworkers have shown that natural and synthetic graphite may contain a series of metals that remain in various proportions after chemical oxidation and reduction to $\mathrm{rGO}^{78,79}$ Among them, the most abundant metals are $\mathrm{Mn}$ and $\mathrm{Fe}$, followed by $\mathrm{Ni}$ and $\mathrm{Cu}$ or $\mathrm{Mo}$ at the level of tens of ppms, but many other elements can be introduced during the synthesis due to the impurity of the chemicals used in large quantities in the preparation of rGO from graphite. ${ }^{79}$ The presence of these metal impurities has been shown to contribute to the electrochemical properties of rGO obtained from graphite. $^{78,79}$

Natural biomass materials may contain, similarly, metals in trace amounts that can be concentrated during the pyrolysis process. Thus, the presence of metal in trace quantities on $\mathrm{G}$ samples appears to be very common and probably unavoidable.

It should be commented, however, that the problem of impurities in catalysis is not exclusive of $\mathrm{G}$ catalysis and that there have been controversial cases in the literature in which the claimed activity of one metal has been disputed ${ }^{81}$ or proved to be due to the presence of impurities of other metals. ${ }^{82}$

There exist several approaches to determine the role of impurities in a catalytic process. One of the most logical ones is to use the purest possible catalysts and to determine the influence of higher purity on the activity. If the activity is not due to impurities, the performance of the catalyst should not decrease. These tests are not easy to be carried out in the case of Gs, due to their difficult purification. 
A second widely used test is based on determination of the nature of the chemical impurities and the evaluation of the catalytic activity of these impurities at the concentrations present as impurity or even somewhat higher. In this case, a safe test consists in adding on purpose increasing amounts of the metal to $\mathrm{G}$ and determining the influence of the presence of increasing concentrations in the range of concentrations present as impurity on the initial reaction rate and final conversion of the impurified sample.

$\mathrm{Mn}$ is generally by large the metal in highest proportion on GO and rGO. This test based on addition of $\mathrm{Mn}$ in hundreds of ppms to $\mathrm{G}$ has been used to rule out the influence of this metal at the percentages present in rGO in the selective acetylene hydrogenation. ${ }^{30}$ Addition of incremental amounts of impurities on the catalyst is better than evaluation of the activity of the impurity in the absence of $\mathrm{G}$, due to the possible synergy that can be established between the impurity and G. From the catalytic point of view the lack of influence of Mn can be easily understood considering the negligible hydrogenation activity of this transition metal, particularly at low concentration.

However, in other cases, such as the activity of Gs as Fenton catalyst, the doubts are more reasonable, particularly considering that $\mathrm{Fe}$ and $\mathrm{Mn}$ are both metals that exhibit under certain conditions high catalytic activity for the Fenton reaction. ${ }^{56}$ Also in this case, it was found that addition of $\mathrm{Mn}$ in various amounts does not change the temporal profile of the reaction, respect to the activity of the G catalyst. 


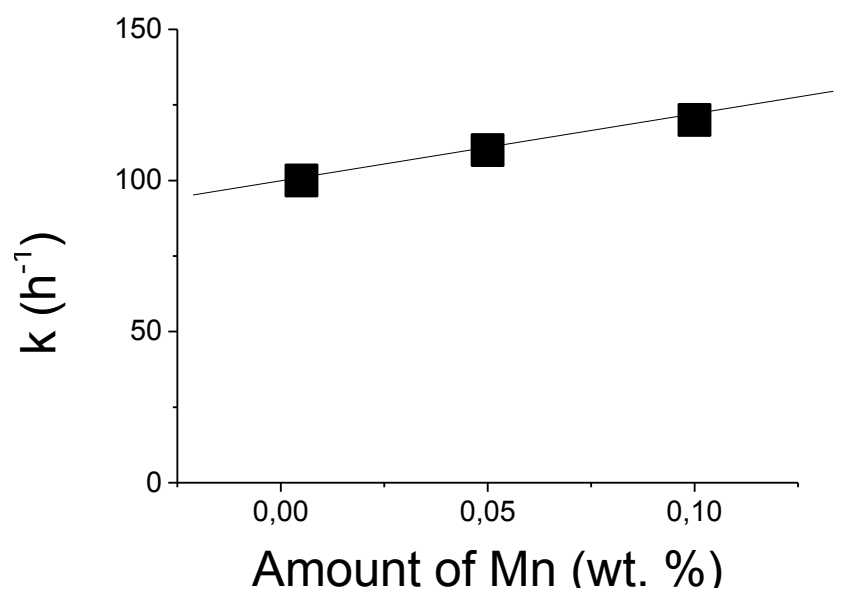

Figure 3. Influence of the amount of $\mathrm{Mn}$ added purposely as impurity to defective $\mathrm{G}$ on initial reaction rate of phenol disappearance. Extrapolation to $0 \%$ indicates that defective $G$ should have an intrinsic catalytic activity when Mn free of about $95 \mathrm{~h}^{-1}$.

\section{7. $\mathrm{N}$ and $\mathrm{B}$ as active sites}

As already commented, pyrolysis of some biopolymers leads to doped Gs. Depending on the pyrolysis temperature, chitosan affords $(\mathrm{N}) \mathrm{G}$ with a maximum $\mathrm{N}$ content of about $7 \mathrm{wt} \%$ that decreases as the pyrolysis temperature increases. Similarly, (B)G can be obtained by pyrolysis of the borate ester of alginate. Other ways to obtain these doped defective Gs can be by reacting GO with ammonia and borax or borate. Doping with heteroelements is a general methodology to modify the properties of Gs, particularly with respect to their activity as carbocatalysts.

In one of the pioneering studies, aerobic oxidation of benzylic alcohols was found to fail to be promoted by $\mathrm{G}$, while $(\mathrm{N}) \mathrm{G}$ catalyzes the reaction. ${ }^{84}$ Furthermore, analysis by XPS of the different families of $\mathrm{N}$ atoms in $\mathrm{G}$ led to the conclusion that the 
activity of $(\mathrm{N}) \mathrm{G}$ correlates with the population of graphenic $\mathrm{N}$ that were proposed to be the active sites for interaction with $\mathrm{O}_{2} \cdot{ }^{84}$ Similarly, in the aerobic oxidation of styrene to a mixture of benzaldehyde and styrene oxide, it was found that $(\mathrm{N}) \mathrm{G}$ from chitosan pyrolysis and (B)G from alginate borate were more active than $G$ derived from pyrolysis of alginate. $^{45}$

Going a step forward, it is clear that co-doping introducing two elements having, in principle, opposing Lewis acid/base activity is a powerful tool to introduce functionality on the $\mathrm{G}$ sheet. Codoping is interesting from the point of view of creating frustrated Lewis acid-base pairs. As already said, theoretical calculations using on layer of (B)G interacting with a second layer of doped $G$ have shown the possibility to activate $\mathrm{H}_{2}$ molecules within the interlayer. ${ }^{36}$ In one of the experimental studies, it was found that $\mathrm{B}$ and $\mathrm{N}$ codoping on defective $\mathrm{G}$ is the most active carbocatalyst compared with $\mathrm{G},(\mathrm{B}) \mathrm{G}$ and $(\mathrm{N}) \mathrm{G}$ for the aerobic oxidation of benzylic hydrocarbons. ${ }^{45}$

It is clear that doping will be further exploited as a general tool for introducing active sites or enhancing the activity of the neighbor sites of Gs as carbocatalysts.

\section{Beyond G catalysis}

In any case, it has to be agreed that the current situation is still somewhat controversial due to the lack of deep understanding on the nature of the active sites for most reactions and some doubts about the possible influence of metals are still reasonable. Determination of the nature of the active sites conclusively will not only rule out metal impurities as the origin of the catalytic activity claimed for Gs, but also will give hints of how to improve the catalytic efficiency of these materials. Surely the wanted evidence of metal-free catalysis is to reproduce the active sites found in Gs in 
synthetic organic molecules, polymers or other materials. One of the major limitations of $\mathrm{G}$ at the present, that hopefully will be overcome in the future, is how to control at atomic level the position and nature of the defects and doping elements. However, until these synthetic tools are developed in a convenient way, Gs have a wide distribution of sites that is against the "single-site" concept of heterogeneous catalysis, according to which ideal heterogeneous catalysts should have exclusively the type of site that is the optimal for a given reaction. ${ }^{85,86}$

This wide distribution of different sites with limited synthetic control in their population and structure is a major drawback of Gs as catalysts and research aimed at overcoming it should be a priority in this area that should be developed in par with the understanding of the nature of the active sites. After disclosure of the wanted sites, it is possible to imagine that post-synthetic modifications of preformed Gs by physical or chemical means could introduce on the ideal $\mathrm{G}$ or in other types of Gs the centers that can promote the target reaction. But also it is possible to design other organocatalysts that must exhibit activity for the target process.

Study of the nature of the active sites has to be made by using simultaneously kinetic studies, deep spectroscopic and microscopic characterization and theoretical calculations. Initially, theory should model the structure of the claimed active sites embedded in the $\mathrm{G}$ sheet and determine reasonable reaction mechanisms with activation barriers. Starting from this point, computational modeling should go beyond and predict the influence that structural parameters can exert on the active site, proposing further modifications on the $\mathrm{G}$ material to render it more active or selective by decreasing the activation energy of the rate determining step. 
It is clear that classical kinetic and catalytic studies are powerful tools that can provide the necessary initial information, leading to reasonable proposals of the structure of the active sites. We have already commented general catalytic experiments used to rule out metal impurities as responsible of the observed catalytic activity in Gs (Figure 3) and now some further controls to determine the structure of active sites will be commented.

Proposals about the structure of the active sites should be based on the knowledge of the composition and functional groups present in G. As an example, in carbon nanotubes (CNTs) there has been found a relationship between the CNT oxygen content and the catalytic activity of the material for the oxidative dehydrogenation of light alkanes into alkenes. ${ }^{87}$ A linear relationship between the catalytic activity for oxidative dehydrogenation of activated carbons and the density of $\mathrm{C}=\mathrm{O}$ groups measured by thermoprogrammed desorption led to propose this type of groups as the active sites for the process. ${ }^{17}$ In the specific case of CNTs, since oxygen in CNT is assumed to be present as hydroxyl, carbonyl and carboxylic acid groups, then, the beneficial influence of the oxygen content on the catalytic activity for this reaction should be related with the presence of any or several of these functional groups. ${ }^{88}$ By contrasting the catalytic activity of pristine CNT with that of modified samples in which selective masking of each of the three types of oxygenated groups was performed, then, evidence in support that the catalytic activity is related to the carbonyl groups was provided. ${ }^{87}$ Scheme 16 illustrates this strategy. Similar conclusions claiming vic-diketo groups or quinone-like structures have been also proposed as active sites of the oxidative dehydrogenation of alkanes in $\mathrm{rGO}^{89}$ It is clear that this type of studies on the influence of selective masking on the catalytic activity provide a useful information and should be performed routinely also for other types of reactions. 


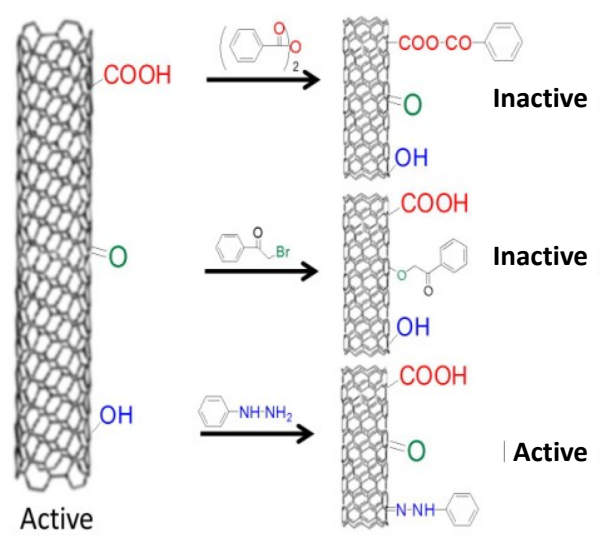

Scheme 16. Selective masking of oxygenated functional groups to determine the nature of the active sites in CNTs for the oxidative dehydrogenation of alkanes. (Taken with permission from ref. ${ }^{87}$ ).

Similar type of selective group masking has led to propose also ketonic groups as the active sites in CNTs for the hydrogenation of nitro groups by hydrazine as reducing agent. ${ }^{90}$ Considering that the ketonic groups in CNT are of quinone-like type, organic chemistry concepts can be used to assess how reasonable the proposal is, by providing examples of similar behavior in structurally related organic molecules or structures.

This type of studies is based on the possibility to apply general organic reactions to the modification of graphenic materials. It should be commented, however, the methodology has to be adapted to the nature of the $G$ material. In this sense, CNT should have, in principle, a low oxygen content in their structure of a few percents. In contrast, GO has much higher oxygen content at about $50 \%$, with other reactive oxygenated functional groups such as epoxides (Scheme 17) and the possibility of complete, selective masking without altering in a deep extent the original structure is considerably more difficult. Thus, for instance, for the previously commented oxidative 
dehydrogenation of light alkanes, GO is also an active catalyst and in this material epoxides have been claimed as the groups responsible for the observed activity. ${ }^{27}$

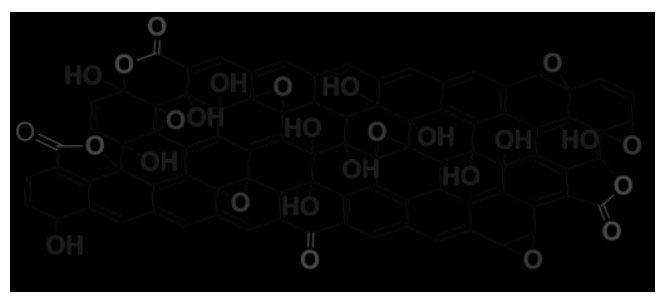

Scheme 17. Proposed model structure of GO that is in accordance with the oxygen content and ${ }^{13} \mathrm{C}$ NMR spectra. (Taken with permission from ref. ${ }^{27}$ )

Alternatively catalytic experiments can be carried out in the presence of additives that mask certain types of sites or introduce new ones and the activity of the additivated catalysts is compared to that of the plain catalyst. As an example, the activity for the selective hydrogenation of acetylene in the presence of a large excess of ethylene of defective $\mathrm{G}$ changes reversibly when $\mathrm{CO}_{2}$ (increasing) or ammonia (decreasing) is added to the feed (Figure 4). ${ }^{30} \mathrm{CO}_{2}$ reacts with basic sites and ammonia neutralizes the acid ones, so the influence in the catalytic activity of $\mathrm{G}$ or $\mathrm{CO}_{2}$ and $\mathrm{NH}_{3}$ is taken as support that basic and acid sites are involved in the reaction mechanism, since otherwise $\mathrm{CO}_{2}$ is an inert gas that should not alter the catalytic activity. Note that the presence of these additives can decrease or increase the intrinsic catalytic activity, something that also agrees with the concept that only a fraction of the total acid and base centers (those that are in the correct distance range) are active to promote the reaction. 


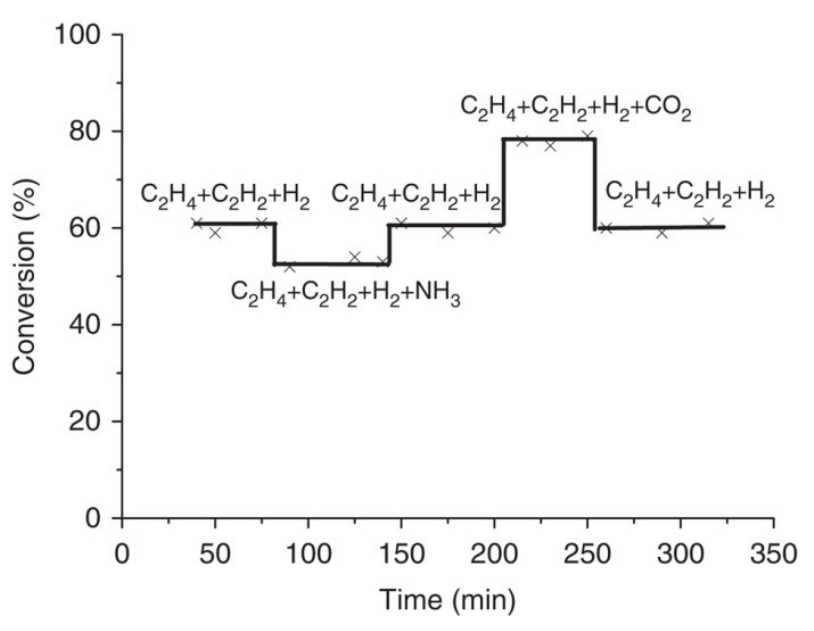

Figure 4. Influence of the presence of $\mathrm{NH}_{3}$ or $\mathrm{CO}_{2}$ in the stream in the gas-phase, continuous-flow selective hydrogenation of acetylene in a large excess of ethylene at $100{ }^{\circ} \mathrm{C}$ (Figure taken with permission from ref. ${ }^{30}$ )

Similarly the involvement of acid sites has been demonstrated in the mechanism of the aqueous phase reforming of glycerol by $\mathrm{N}$ and B-codoped $\mathrm{G}$, by observing the influence that triarylborane in $1 \mathrm{wt} \%$ respect to $\mathrm{G}$ plays in glycerol conversion and selectivity towards $\mathrm{H}_{2}$ production (Figure 5). ${ }^{91}$

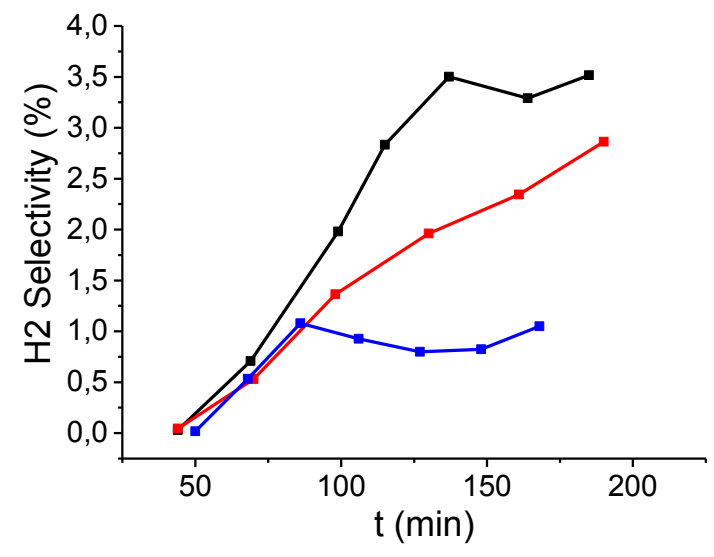

Figure 5. Influence on the activity of defective $\mathrm{G}$ for the aqueous phase reforming of glycerol of the addition of $1 \mathrm{wt} \%$ of an organoboron molecule as Lewis acid. Black line: G plus 1wt $\%$ tris(pentafluorophenyl)borane; Red line: G plus $1 \mathrm{wt} \%$ of 
bis(pinacoltl)diborane; Blue line: $\mathrm{G}$ without additive. Reaction conditions: $3.43 \mathrm{mmol}$ glycerol (10 vol./vol\% glycerol/water), $20 \mathrm{wt} \%$ catalyst, $\mathrm{P}_{0}=1$ bar.

Characterization techniques are also extremely valuable to gain insight into the nature of the active sites. Electron microscopy can reveal the presence of holes on $G$ that may increase significantly the periphery of the $G$ sheet. Characterization by electron microscopy has also served to propose the nature of active sites on G. In one of these studies aimed at disclosing the nature of the active sites in GO to promote the aerobic oxidation of benzylamines to the corresponding N-benzylidene benzylamine, Loh and coworkers observed by electron microscopy that GO obtained by Hummers oxidation contains amorphous debris that can be removed from the rGO sample by acid washings and subsequent neutralization (Scheme 18). ${ }^{9}$ Since Hummers oxidation of graphite is a harsh oxidative treatment it is reasonable that, as in the case of GO, acid carboxylic groups are formed even in the small amorphous carbon NPs formed in the process. These carboxylic acid groups can be used to purify GO from these unwanted carbon NPs by washing with base. Interestingly, removal of these oxidized amorphous particles makes available free holes on the GO sheet as observed by TEM and this makes active the material as oxidation catalyst. Overall, this TEM characterization provides evidence in support of sheet holes as the active sites of the reaction. 


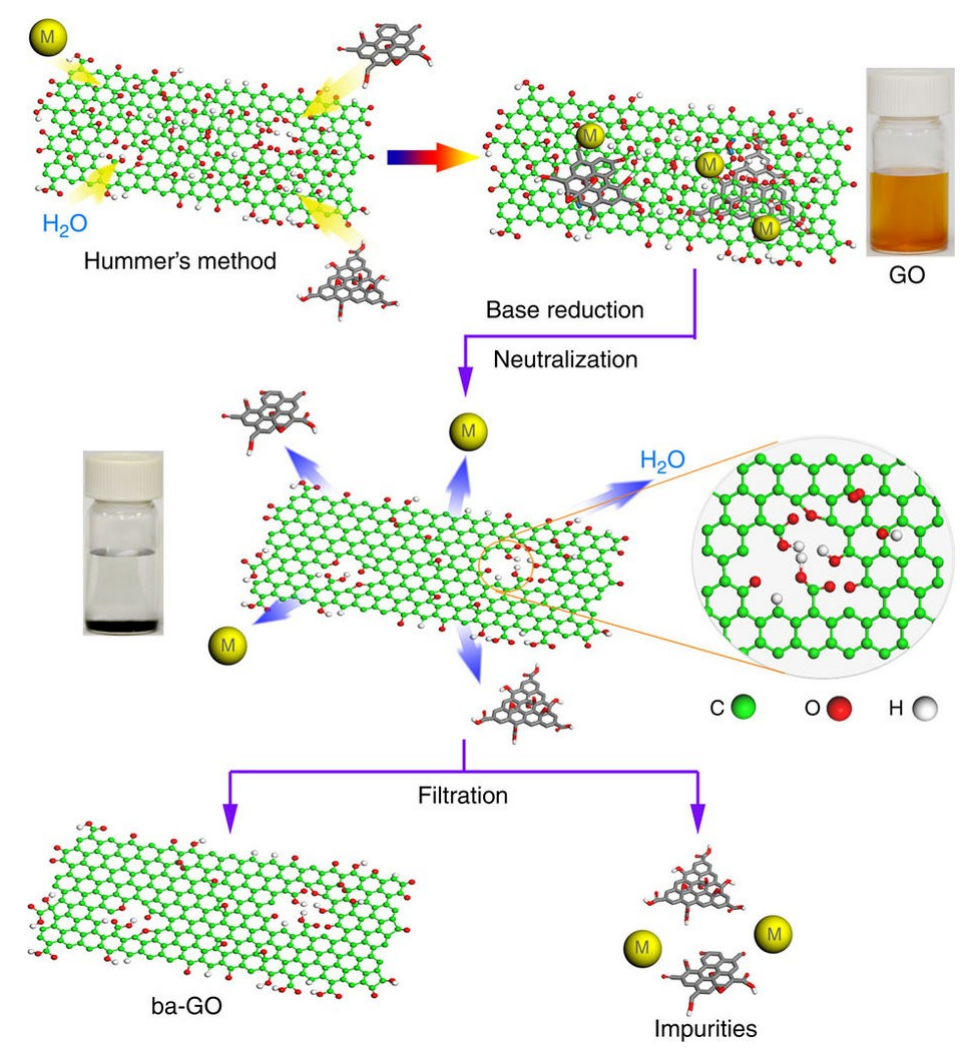

Scheme 18. Purification process of GO by base and acid washings to release free holes from amorphous impurities resulting in an increase in its catalytic activity (taken with permission from ref. ${ }^{9}$ ).

While IR spectroscopy is very useful for GO characterization, the information provided by this technique for $\mathrm{rGO}$ and Gs in general is of lower value, among other reasons due to lack of transparency of these materials that do not generally allow applying transmission techniques. However, other techniques based on reflectance could be adequate. IR spectroscopy has been very useful to characterize species adsorbed on the surface of solids and to determine in situ their evolution upon controlled admission of reagents. It is clear that future research on $\mathrm{G}$ would be much facilitated by this type of mechanistic studies. As already commented in one particular case, the nature of the active sites on GO for the oxidation of amines to nitriles by hypochlorite as oxidizing reagent has been proposed to be carboxylic acids based on the 
IR spectral changes. In the process it is claimed that carboxylic acids will be converted into peroxyacids or peroxylactones by $\mathrm{ClO}^{-}$and these highly reactive carboxylic acid derivatives will be responsible for the conversion of the amines to nitriles (Scheme 10). Considering that GO and other $\mathrm{G}$ materials have a considerable population of carboxylic acid groups mainly at the periphery and holes of the sheet, it can be foreseen that in the near future Gs could be used as metal-free catalyst for those processes that can be promoted by peracids such as the alkene epoxidations, Bayer-Villiger oxidative rearrangement of cyclic ketones and sulfide oxidation, among others. ${ }^{54}$

\section{From Gs to organocatalysis.}

Most of the centers that are presumed as exhibiting catalytic activity in Gs can also be found in organic molecules that should be also active for the process. Organocatalysis should start at this point trying to increase the stability and the activity of the organic molecules by applying organic concepts such as introduction of electron donor or withdrawing substituents, tuning steric hindrance around the active group and preparation of derivatives, among others. Then, next steps could be to anchor these molecules on large surface area supports trying to transform homogeneous catalysts into heterogeneous and recoverable systems. Alternatively these molecules can be encapsulated into inert matrices to protect them or to prepare soluble and insoluble polymers having these active centers.

Besides organic synthesis and easy derivatization, organocatalysis has the advantage over carbocatalysis that the former can, in principle, prepare single site catalysts and materials having a larger density of sites than those that can be obtained in Gs. On the other way around, the knowledge gained in organocatalysis can serve for the 
initial proposal of the nature of active sites and for the post-synthetic modification of Gs increasing their performance.

Probably, one of the main limitations in transferring catalysis by Gs to organocatalytic processes is when the active sites are carbon vacancies and holes. But also in this case, rigid large aromatic molecules having persistent radicals or biradical centers can be envisioned to emulate the performance of Gs.

\section{Future directions in carbocatalysis by Gs.}

The field of Gs as carbocatalysts is certainly in its infancy and can be considered that started in 2010 by Bielawski's findings on the catalytic activity of graphite oxide and GO. ${ }^{42,43}$ This short period of time with the limited effort that has been made so far, together with the growing pressure for replacing critical metals, makes us anticipate that the field will attract an increasing interest of researchers in the area of catalysis in the next years to come. One of the clear targets is to delineate for which reactions, Gs can be catalysts with comparable activity with respect to other alternatives.

At the moment, Gs have well-proven catalytic activity to promote some aerobic oxidations, while information about other reaction types is more limited. In principle the objective is to show that most of the reactions that are promoted by metals can also be catalyzed by Gs. Metals can act as Lewis acid sites and as redox centers and, as commented, these type of centers can also be present in Gs. Clear objectives of Gs is to show their activity in catalytic reactions that are supposed to be carried out exclusively by metal-containing catalysts. Typical reactions catalyzed by metals are reductions, including hydrogenation, oxidations, Lewis acid-promoted processes, rearrangements and homo and cross couplings, Examples of Gs as metal-free catalysts for reductions and oxidations are also sufficiently documented. ${ }^{27}$ However, in spite of their large 
importance in modern organic synthesis, the activity of $\mathrm{G}$ in coupling reactions has not been reported so far. In principle, based on the most widely accepted mechanism for Pd catalysis of this reaction, the active centers should be able to insert into aryl-halide bonds through oxidative addition and subsequently undergo transmetallation and reductive elimination (Scheme 19). Since, as discussed earlier, redox centers are available on $\mathrm{G}$, it seems that the key point is to have on the $\mathrm{G}$ sheet a functional group or defect able to insert into the strong aryl-halide bond. It should be commented that, as Corma has shown ${ }^{92,93}$ in the case of Au NPs, the main difference with homogeneous Pd catalysis can be that in the case of $\mathrm{G}$ the mechanism can be performed by using more than one atom or center and, therefore, it can be envisioned that the combination of electrophilic defects with redox sites could somehow act as a single $\mathrm{Pd}$ atom in a molecular complex (Scheme 20). If this were the case, then, most of the widely used CC bond formation cross coupling reaction could be promoted by Gs.

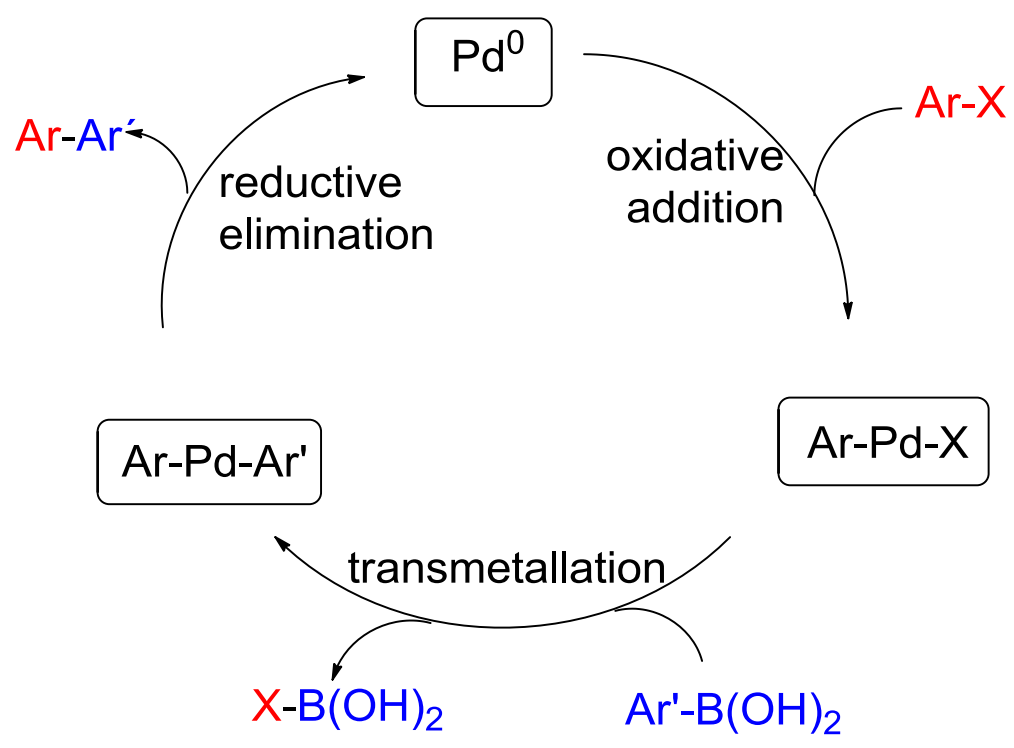

Scheme 19. Commonly accepted steps in the Pd catalyzed Suzuki-Miyaura coupling. 


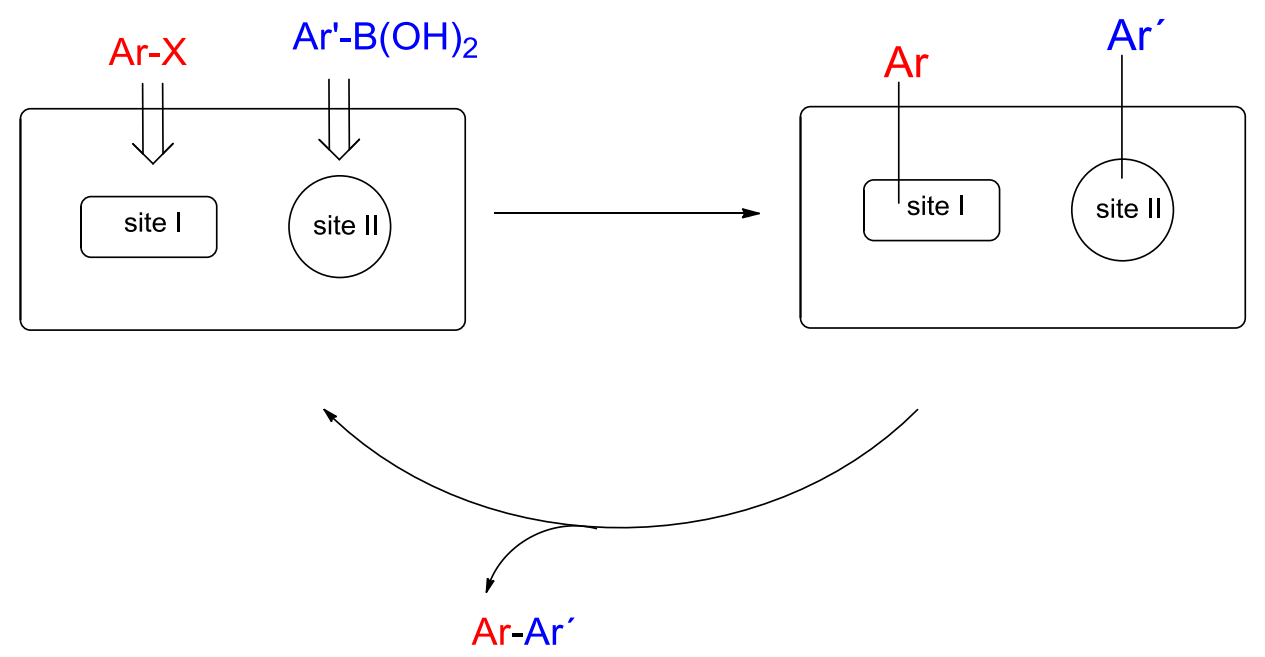

Scheme 20. Proposal for catalytic cross-coupling based on a metal-free catalyst the combination of two sites with different properties rather than a single $\mathrm{Pd}$ atom can promote the coupling reaction.

Besides delineating for which reactions Gs can be catalysts, disclosure of the corresponding active sites and the two-way interplay with organocatalysis is now mandatory for every reaction catalyzed by carbons. Comparison of the activity of various Gs in which oxygenated functional groups have been selectivity masked should be performed as a general tool to address the role of these groups in catalysis. It is clear that the final target is to use Gs as metal-free catalyst in a large scale industrial process. Development of metal-free $G$ catalysis for an industrial process would mean that the combination of properties and availability of a certain $G$ would make this material preferable as catalyst to any other alternatives, including those based on metals and, surely, will trigger much more effort trying to expand the use of Gs to other related large-scale process.

In this context, it is difficult to envision Gs as replacement of noble metals in already-existing, well-established industrial processes, where a change in the catalyst would require massive capital investments and the technology has been already paid 
back. However, Gs can have their opportunity in new areas that are currently being developed and that are close to commercial implementation, but still are searching for the most advantageous catalyst. It appears to us that biomass conversion and novel power-to-chemicals reactions in the context of renewable energies are more suitable fields to evaluate Gs as metal-free catalysts in comparison with metal-containing catalysts. For instance, one of the common problems in biomass conversion is the formation of heavy tars and the difficulty in recovering and reusing the catalyst. If the catalyst would be based on G obtained in an affordable way, it could be envisioned that it could not be necessary to separate the catalyst from the final wastes, since after its use it could be processed in the residue as an additional carbonaceous component of the mixture. As already commented, there are certain biomass processes such as cellulose hydrolysis currently carried out by homogeneous acids that can take place also with acidic rGOs. Similarly, glucose isomerization and dehydration or the direct glucose conversion to succinic acid are processes that can be promoted by modified Gs. Also, in power-to-chemicals in which conversion of renewable electricity to chemicals is going to become highly important, there are electrolytic processes in which Gs can act as electrocatalysts, increasing the efficiency of the electrochemical process and avoiding or minimizing the amount of metal present in the electrode.

Thus, the final summary of this Perspective article is that metal-free catalysis by G will be intensely researched in the next years and that the outcome of this research could be a revolutionary change in the field of catalysis, moving from metal catalysis that has predominated since the early days of catalysis up to the XXth Century to a sustainable catalysis based on the use of carbon atoms in which the centers can be tuned by applying concepts of organic chemistry and, hopefully, engineered at atomic level. 
Deep understanding of the active sites in $\mathrm{G}$ will lead to a unified view of carbocatalysis and organocatalysis.

\section{Acknowledgements.}

Financial support by the Spanish Ministry of Economy and Competitiveness (Severo Ochoa, Grapas and CTQ2015-69153-CO2-1) and Generalitat Valenciana (Prometo 2013-014) is gratefully acknowledged. A.P. also thanks the Spanish Ministry of Economy and Competitiveness for a Ramón y Cajal research associate contract.

\section{References.}

1. Kroschwitz, J. I.; Howe-Grant, M.; Siegel, P. M.; Richman, B.; E., Z.; Altieri, L., KirkOthmer Encyclopedia of Chemical Technology. Wiley: New York, 1999.

2. Bullock, R. M., Catalysis without Precious Metals. Wiley: New York, 2010.

3. Commission, E. Critical Raw Materials.

4. Oliver-Meseguer, J.; Cabrero-Antonino, J. R.; Dominguez, I.; Leyva-Perez, A.; Corma, A., Small Gold Clusters Formed in Solution Give Reaction Turnover Numbers of 10(7) at Room Temperature. Science 2012, 338 (6113), 1452-1455.

5. Yang, X.-F.; Wang, A.; Qiao, B.; Li, J.; Liu, J.; Zhang, T., Single-Atom Catalysts: A New Frontier in Heterogeneous Catalysis. Accounts of Chemical Research 2013, 46 (8), 1740-1748.

6. Abbet, S.; Sanchez, A.; Heiz, U.; Schneider, W. D.; Ferrari, A. M.; Pacchioni, G.; Rosch, $\mathrm{N}$., Acetylene cyclotrimerization on supported size-selected Pd-n clusters $(1<=n<=30)$ : one atom is enough! Journal of the American Chemical Society 2000, 122 (14), 3453-3457.

7. Corma, A.; Concepcion, P.; Boronat, M.; Sabater, M. J.; Navas, J.; Yacaman, M. J.; Larios, E.; Posadas, A.; Arturo Lopez-Quintela, M.; Buceta, D.; Mendoza, E.; Guilera, G.; Mayoral, A., Exceptional oxidation activity with size-controlled supported gold clusters of low atomicity. Nature Chemistry 2013, 5 (9), 775-781.

8. $\quad$ Lange, K.; Visser, B.; Neuwirth, D.; Eckhard, J. F.; Boesl, U.; Tschurl, M.; Bowen, K. H.; Heiz, U., Size-dependent gas phase reactivity of tantalum cluster cations with small alcohols. International Journal of Mass Spectrometry 2015, 375, 9-13.

9. $\quad$ Su, C.; Acik, M.; Takai, K.; Lu, J.; Hao, S.-j.; Zheng, Y.; Wu, P.; Bao, Q.; Enoki, T.; Chabal, Y. J.; Ping Loh, K., Probing the catalytic activity of porous graphene oxide and the origin of this behaviour. Nat Commun 2012, 3, 1298.

10. Zhang, J.; Su, D.; Zhang, A.; Wang, D.; Schloegl, R.; Hebert, C., Nanocarbon as robust catalyst: Mechanistic insight into carbon-mediated catalysis. Angewandte ChemieInternational Edition 2007, 46 (38), 7319-7323. 
11. Primo, A.; Atienzar, P.; Sanchez, E.; Maria Delgado, J.; Garcia, H., From biomass wastes to large-area, high-quality, $\mathrm{N}$-doped graphene: catalyst-free carbonization of chitosan coatings on arbitrary substrates. Chemical Communications 2012, 48 (74), 9254-9256.

12. Primo, A.; Forneli, A.; Corma, A.; Garcia, H., From Biomass Wastes to Highly Efficient CO2 Adsorbents: Graphitisation of Chitosan and Alginate Biopolymers. Chemsuschem 2012, 5 (11), 2207-2214.

13. Jüntgen, H., Activated carbon as catalyst. ChemInform 1987, 18 (19).

14. Singoredjo, L.; Kapteijn, F.; Moulijn, J. A.; MartÃ-n-MartÃ-nez, J.-M.; Boehm, H.-P., Modified activated carbons for the selective catalytic reduction of NO with NH3. Carbon 1993, 31 (1), 213-222.

15. Bagreev, A.; Bashkova, S.; Bandosz, T.; Menendez, A.; Dukhno, I.; Tarasenko, Y., Nitrogen Enriched Activated Carbons as Adsorbents and Catalysts in Desulfurization Technologies. Prepr. Pap.-Am. Chem. Soc., Div. Fuel Chem 2004, 49 (2), 920.

16. Larsen, E. C.; Walton, J. H., Activated Carbon as a Catalyst in Certain OxidationReduction Reactions. The Journal of Physical Chemistry 1940, 44 (1), 70-85.

17. Pereira, M. F. R.; Ã“rfãfo, J. J. M.; Figueiredo, J. L., Oxidative dehydrogenation of ethylbenzene on activated carbon catalysts. I. Influence of surface chemical groups. Applied Catalysis A: General 1999, 184 (1), 153-160.

18. Su, D. S.; Perathoner, S.; Centi, G., Nanocarbons for the Development of Advanced Catalysts. Chemical Reviews 2013, 113 (8), 5782-5816.

19. Zhang, J.; Su, D. S.; Blume, R.; Schlögl, R.; Wang, R.; Yang, X.; Gajovia, A., Surface Chemistry and Catalytic Reactivity of a Nanodiamond in the Steamâ€国Free Dehydrogenation of Ethylbenzene. Angewandte Chemie International Edition 2010, 49 (46), 8640-8644.

20. Tasis, D.; Tagmatarchis, N.; Bianco, A.; Prato, M., Chemistry of carbon nanotubes. Chemical Reviews 2006, 106 (3), 1105-1136.

21. Pereira, M. F. R.; Figueiredo, J. L.; Orfeo, J. J.; Serp, P.; Kalck, P.; Kihn, Y., Catalytic activity of carbon nanotubes in the oxidative dehydrogenation of ethylbenzene. Carbon 2004, 42 (14), 2807-2813.

22. Huang, Y.; Wang, Y.; Zhao, R.; Shen, P. K.; Wei, Z., Accurately measuring the hydrogen generation rate for hydrolysis of sodium borohydride on multiwalled carbon nanotubes/Coâ€"B catalysts. international journal of hydrogen energy 2008, 33 (23), 71107115.

23. Serp, P.; Corrias, M.; Kalck, P., Carbon nanotubes and nanofibers in catalysis. Applied Catalysis A: General 2003, 253 (2), 337-358.

24. Wang, Y.; Wei, F.; Luo, G.; Yu, H.; Gu, G., The large-scale production of carbon nanotubes in a nano-agglomerate fluidized-bed reactor. Chemical Physics Letters 2002, 364 (5), 568-572.

25. Landi, B. J.; Ganter, M. J.; Cress, C. D.; DiLeo, R. A.; Raffaelle, R. P., Carbon nanotubes for lithium ion batteries. Energy \& Environmental Science 2009, 2 (6), 638-654.

26. Kong, X.-K.; Chen, C.-L.; Chen, Q.-W., Doped graphene for metal-free catalysis. Chemical Society Reviews 2014, 43 (8), 2841-2857.

27. Navalon, S.; Dhakshinamoorthy, A.; Alvaro, M.; Garcia, H., Carbocatalysis by Graphene-Based Materials. Chemical Reviews 2014, 114 (12), 6179-6212.

28. Albero, J.; Garcia, H., Doped graphenes in catalysis. Journal of Molecular Catalysis aChemical 2015, 408, 296-309.

29. Su, D.; Wen, G.; Wu, S.; Peng, F.; Schloegl, R., Carbocatalysts in Liquid Phase. Angewandte Chemie International Edition 2016, doi 10.1002/anie.201600906.

30. Primo, A.; Neatu, F.; Florea, M.; Parvulescu, V.; Garcia, H., Graphenes in the absence of metals as carbocatalysts for selective acetylene hydrogenation and alkene hydrogenation. Nature Communications 2014, 5.

31. Pawar, S. N.; Edgar, K. J., Alginate derivatization: A review of chemistry, properties and applications. Biomaterials 2012, 33 (11), 3279-3305. 
32. Primo, A.; Sanchez, E.; Delgado, J. M.; García, H., High-yield production of N-doped graphitic platelets by aqueous exfoliation of pyrolyzed chitosan. 2014; Vol. 68, pp 777-783.

33. Trandafir, M.-M.; Florea, M.; Neaţu, F.; Primo, A.; Parvulescu, V. I.; García, H., Graphene from Alginate Pyrolysis as a Metal-Free Catalyst for Hydrogenation of Nitro Compounds. Chemsuschem 2016, 9 (13), 1565-1569.

34. Stephan, D. W.; Erker, G., Frustrated Lewis Pair Chemistry: Development and Perspectives. Angewandte Chemie International Edition 2015, 54 (22), 6400-6441.

35. Chernichenko, K.; Nieger, M.; Leskela, M.; Repo, T., Hydrogen activation by 2-boryl$\mathrm{N}, \mathrm{N}$-dialkylanilines: a revision of Piers' ansa-aminoborane. Dalton Transactions 2012, 41 (30), 9029-9032.

36. Sun, X.; Li, B.; Liu, T.; Song, J.; Su, D. S., Designing graphene as a new frustrated Lewis pair catalyst for hydrogen activation by co-doping. Physical Chemistry Chemical Physics 2016, 18 (16), 11120-11124.

37. Diño, W. A.; Miura, Y.; Nakanishi, H.; Kasai, H.; Sugimoto, T.; Kondo, T., H2 dissociative adsorption at the armchair edges of graphite. Solid State Communications 2004, 132 (10), 713-718.

38. Diño, W. A.; Nakanishi, H.; Kasai, H.; Sugimoto, T.; Kondo, T., H<sub $>2</$ sub $>$ Dissociative Adsorption at the Zigzag Edges of Graphite. e-Journal of Surface Science and Nanotechnology 2004, 2, 77-80.

39. Allouche, A.; Ferro, Y., Dissociative adsorption of small molecules at vacancies on the graphite (0 00 1) surface. Carbon 2006, 44 (15), 3320-3327.

40. Jiang, Q. G.; Ao, Z. M.; Zheng, W. T.; Li, S.; Jiang, Q., Enhanced hydrogen sensing properties of graphene by introducing a mono-atom-vacancy. Physical Chemistry Chemical Physics 2013, 15 (48), 21016-21022.

41. IUPAC, Compendium of Chemical Terminology, (the "Gold Book"). 2nd ed.; Blackwell Scientific Publications: Oxford, 1997.

42. Dreyer, D. R.; Bielawski, C. W., Carbocatalysis: Heterogeneous carbons finding utility in synthetic chemistry. Chemical Science 2011, 2 (7), 1233-1240.

43. Dreyer, D. R.; Jia, H.-P.; Bielawski, C. W., Graphene Oxide: A Convenient Carbocatalyst for Facilitating Oxidation and Hydration Reactions. Angewandte ChemieInternational Edition 2010, 49 (38), 6813-6816.

44. Dreyer, D. R.; Jia, H.-P.; Todd, A. D.; Geng, J.; Bielawski, C. W., Graphite oxide: a selective and highly efficient oxidant of thiols and sulfides. Organic \& Biomolecular Chemistry 2011, 9 (21), 7292-7295.

45. Dhakshinamoorthy, A.; Primo, A.; Concepcion, P.; Alvaro, M.; Garcia, H., Doped Graphene as a Metal-Free Carbocatalyst for the Selective Aerobic Oxidation of Benzylic Hydrocarbons, Cyclooctane and Styrene. Chemistry-a European Journal 2013, 19 (23), 75477554.

46. Jia, H.-P.; Dreyer, D. R.; Bielawski, C. W., C-H oxidation using graphite oxide. Tetrahedron 2011, 67 (24), 4431-4434.

47. Jia, H.-P.; Dreyer, D. R.; Bielawski, C. W., Graphite Oxide as an Auto-Tandem Oxidation-Hydration-Aldol Coupling Catalyst. Advanced Synthesis \& Catalysis 2011, 353 (4), 528-532.

48. Dhakshinamoorthy, A.; Latorre-Sanchez, M.; Asiri, A. M.; Primo, A.; Garcia, H., Sulphur-doped graphene as metal-free carbocatalysts for the solventless aerobic oxidation of styrenes. Catalysis Communications 2015, 65, 10-13.

49. Boukhvalov, D. W.; Dreyer, D. R.; Bielawski, C. W.; Son, Y.-W., A Computational Investigation of the Catalytic Properties of Graphene Oxide: Exploring Mechanisms by using DFT Methods. Chemcatchem 2012, 4 (11), 1844-1849.

50. Onda, A.; Ochi, T.; Yanagisawa, K., Selective hydrolysis of cellulose into glucose over solid acid catalysts. Green Chemistry 2008, 10 (10), 1033-1037.

51. V. I.Parvulescu and H. Garcia, manuscript under preparation. 
52. Blandez, J. F.; Navalon, S.; Alvaro, M.; Garcia, H., Graphenes as Metal-free Catalysts for the Oxidative Depolymerization of Lignin Models. Chemcatchem 2015, 7 (18), 3020-3026.

53. Primo, A.; Puche, M.; Pavel, O. D.; Cojocaru, B.; Tirsoaga, A.; Parvulescu, V.; Garcia, H., Graphene oxide as a metal-free catalyst for oxidation of primary amines to nitriles by hypochlorite. Chemical Communications 2016, 52 (9), 1839-1842.

54. Smith, M. B.; March, J., March's Advanced Organic Chemistry: Reactions, Mechanisms, and Structure. 6th ed.; John Wiley \& Sons: New York, 2006.

55. Navalon, S.; Alvaro, M.; Garcia, H., Heterogeneous Fenton catalysts based on clays, silicas and zeolites. Applied Catalysis B-Environmental 2010, 99 (1-2), 1-26.

56. Dhakshinamoorthy, A.; Navalon, S.; Alvaro, M.; Garcia, H., Metal Nanoparticles as Heterogeneous Fenton Catalysts. Chemsuschem 2012, 5 (1), 46-64.

57. Navalon, S.; Dhakshinamoorthy, A.; Alvaro, M.; Garcia, H., Heterogeneous Fenton Catalysts Based on Activated Carbon and Related Materials. Chemsuschem 2011, 4 (12), 1712-1730.

58. Aguilar, C.; Garcíale, R.; Soto-Garrido, G.; Arriagada, R. N., Catalytic wet air oxidation of aqueous ammonia with activated carbon. Applied Catalysis B: Environmental 2003, 46 (2), 229-237.

59. Quintanilla, A.; Casas, J. A.; Rodriguez, J. J., Hydrogen peroxide-promoted-CWAO of phenol with activated carbon. Applied Catalysis B: Environmental 2009, 93 (3â€"4), 339-345.

60. Santiago, M.; StÃ $1 / 4$ ber, F.; Fortuny, A. Ì.; Fabregat, A.; Font, J., Modified activated carbons for catalytic wet air oxidation of phenol. Carbon 2005, 43 (10), 2134-2145.

61. Garcia, H., Allotropic Carbon Nanoforms as Advanced Metal-Free Catalysts or as Supports. Advances in Chemistry 2014, 2014, 20.

62. Santos, V. P.; Pereira, M. F. R.; Faria, P. C. C.; Ã “rfÃfo, J. J. M., Decolourisation of dye solutions by oxidation with $\mathrm{H} 2 \mathrm{O} 2$ in the presence of modified activated carbons. Journal of Hazardous Materials 2009, 162 (2â€“3), 736-742.

63. Sánchez-Polo, M.; Salhi, E.; Rivera-Utrilla, J.; von Gunten, U., Combination of Ozone with Activated Carbon as an Alternative to Conventional Advanced Oxidation Processes. Ozone Science \& Engineering Journal 2006, 28, 237 - 245.

64. Duan, X.; Ao, Z.; Sun, H.; Zhou, L.; Wang, G.; Wang, S., Insights into N-doping in single-walled carbon nanotubes for enhanced activation of superoxides: a mechanistic study. Chemical Communications 2015, 51 (83), 15249-15252.

65. Espinosa, J. C.; Navalon, S.; Primo, A.; Moral, M.; Fernandez Sanz, J.; Alvaro, M.; Garcia, H., Graphenes as Efficient Metal-Free Fenton Catalysts. Chemistry-a European Journal 2015, 21 (34), 11966-11971.

66. Espinosa, J. C.; Navalón, S.; Álvaro, M.; García, H., Reduced Graphene Oxide as a Metal-Free Catalyst for the Light-Assisted Fenton-Like Reaction. Chemcatchem 2016, doi $10.1002 /$ cctc. 201600364.

67. Ho, T.-L.; Fieser, M.; Fieser, L.; Danheiser, R.; Roush, W.; Smith, J.; Fieser, L. F., 2,3Dichloro-5,6-dicyano-1,4-benzoquinone (DDQ). In Fieser and Fieser's Reagents for Organic Synthesis, John Wiley \& Sons, Inc.: 2006.

68. Su, D. S.; Zhang, J.; Frank, B.; Thomas, A.; Wang, X.; Paraknowitsch, J.; Schlögl, R., Metal-Free Heterogeneous Catalysis for Sustainable Chemistry. Chemsuschem 2010, 3 (2), 169-180.

69. Dhakshinamoorthy, A.; Alvaro, M.; Puche, M.; Fornes, V.; Garcia, H., Graphene Oxide as Catalyst for the Acetalization of Aldehydes at Room Temperature. Chemcatchem 2012, 4 (12), 2026-2030.

70. Dhakshinamoorthy, A.; Alvaro, M.; Concepcion, P.; Fornes, V.; Garcia, H., Graphene oxide as an acid catalyst for the room temperature ring opening of epoxides. Chemical Communications 2012, 48 (44), 5443-5445. 
71. Zhao, X.; Wang, J.; Chen, C.; Huang, Y.; Wang, A.; Zhang, T., Graphene oxide for cellulose hydrolysis: how it works as a highly active catalyst? Chemical Communications 2014, 50 (26), 3439-3442.

72. Stankovich, S.; Dikin, D. A.; Piner, R. D.; Kohlhaas, K. A.; Kleinhammes, A.; Jia, Y.; Wu, Y.; Nguyen, S. T.; Ruoff, R. S., Synthesis of graphene-based nanosheets via chemical reduction of exfoliated graphite oxide. Carbon 2007, 45 (7), 1558-1565.

73. Garg, B.; Bisht, T.; Ling, Y.-C., Graphene-Based Nanomaterials as Heterogeneous Acid Catalysts: A Comprehensive Perspective. Molecules 2014, 19 (9), 14582-14614.

74. Seredych, M.; Tamashausky, A. V.; Bandosz, T. J., Graphite Oxides Obtained from Porous Graphite: The Role of Surface Chemistry and Texture in Ammonia Retention at Ambient Conditions. Advanced Functional Materials 2010, 20 (10), 1670-1679.

75. Wang, H.; Kong, Q.; Wang, Y.; Deng, T.; Chen, C.; Hou, X.; Zhu, Y., Graphene Oxide Catalyzed Dehydration of Fructose into 5-Hydroxymethylfurfural with Isopropanol as Cosolvent. Chemcatchem 2014, 6 (3), 728-732.

76. Podolean, I.; Anita, F.; García, H.; Parvulescu, V. I.; Coman, S. M., Efficient magnetic recoverable acid-functionalized-carbon catalysts for starch valorization to multiple biochemicals. Catalysis Today 2016, doi:10.1016/j.cattod.2016.07.007.

77. Navalon, S.; Dhakshinamoorthy, A.; Alvaro, M.; Garcia, H., Metal nanoparticles supported on two-dimensional graphenes as heterogeneous catalysts. Coordination Chemistry Reviews 2016, 312, 99-148.

78. Ambrosi, A.; Chua, C. K.; Khezri, B.; Sofer, Z. k.; Webster, R. D.; Pumera, M., Chemically reduced graphene contains inherent metallic impurities present in parent natural and synthetic graphite. Proceedings of the National Academy of Sciences 2012, 109 (32), 12899-12904.

79. Wong, C. H. A.; Sofer, Z.; Kubeaova, M.; KuÄ?lera, J.; Matajkova, S.; Pumera, M., Synthetic routes contaminate graphene materials with a whole spectrum of unanticipated metallic elements. Proceedings of the National Academy of Sciences 2014, 111 (38), 1377413779.

80. Ballesteros-Garrido, R.; Baldovi, H. G.; Latorre-Sanchez, M.; Alvaro, M.; Garcia, H., Photocatalytic hydrogen generation from water-methanol mixtures using halogenated reconstituted graphenes. Journal of Materials Chemistry A 2013, 1 (38), 11728-11734.

81. Corma, A.; Juarez, R.; Boronat, M.; Sanchez, F.; Iglesias, M.; Garcia, H., Gold catalyzes the Sonogashira coupling reaction without the requirement of palladium impurities. Chemical Communications 2011, 47 (5), 1446-1448.

82. Thome, I.; Nijs, A.; Bolm, C., Trace metal impurities in catalysis. Chemical Society Reviews 2012, 41 (3), 979-987.

83. Buchwald, S. L.; Bolm, C., On the Role of Metal Contaminants in Catalyses with FeCl3. Angewandte Chemie-International Edition 2009, 48 (31), 5586-5587.

84. Long, J.; Xie, X.; Xu, J.; Gu, Q.; Chen, L.; Wang, X., Nitrogen-Doped Graphene Nanosheets as Metal-Free Catalysts for Aerobic Selective Oxidation of Benzylic Alcohols. ACS Catalysis 2012, 2 (4), 622-631.

85. Coates, G. W., Precise control of polyolefin stereochemistry using single-site metal catalysts. Chemical Reviews 2000, 100 (4), 1223-1252.

86. Corma, A.; Garcia, H.; Xamena, F., Engineering Metal Organic Frameworks for Heterogeneous Catalysis. Chemical Reviews 2010, 110 (8), 4606-4655.

87. Qi, W.; Liu, W.; Zhang, B.; Gu, X.; Guo, X.; Su, D., Oxidative Dehydrogenation on Nanocarbon: Identification and Quantification of Active Sites by Chemical Titration. Angewandte Chemie-International Edition 2013, 52 (52), 14224-14228.

88. Qi, W.; Su, D., Metal-Free Carbon Catalysts for Oxidative Dehydrogenation Reactions. ACS Catalysis 2014, 4 (9), 3212-3218. 
89. Dathar, G. K. P.; Tsai, Y.-T.; Gierszal, K.; Xu, Y.; Liang, C.; Rondinone, A. J.; Overbury, S. H.; Schwartz, V., Identifying Active Functionalities on Few-Layered Graphene Catalysts for Oxidative Dehydrogenation of Isobutane. Chemsuschem 2014, 7 (2), 483-491.

90. Gu, X.; Qi, W.; Wu, S.; Sun, Z.; Xu, X.; Su, D., Noncovalent functionalization of multiwalled carbon nanotubes as metal-free catalysts for the reduction of nitrobenzene. Catalysis Science \& Technology 2015, 4 (6), 1730-1733.

91. Esteve-Adell, I.; Primo, A.; GarcÃa, H., unprublished results.

92. Boronat, M.; Combita, D.; ConcepciÃ ${ }^{3}$, P.; Corma, A.; Garcia, H.; Juarez, R.; Laursen, S.; de Dios López-Castro, J., Making Câ€“C Bonds with Gold: Identification of Selective Gold Sites for Homo- and Cross-Coupling Reactions between lodobenzene and Alkynes. The Journal of Physical Chemistry C 2012, 116 (47), 24855-24867.

93. Boronat, M.; Lopez-Ausens, T.; Corma, A., Making Câ€"C Bonds with Gold Catalysts: A Theoretical Study of the Influence of Gold Particle Size on the Dissociation of the Câ $€^{\text {" } X}$ Bond in Aryl Halides. The Journal of Physical Chemistry C 2014, 118 (17), 9018-9029. 
Table of Contents Graphic

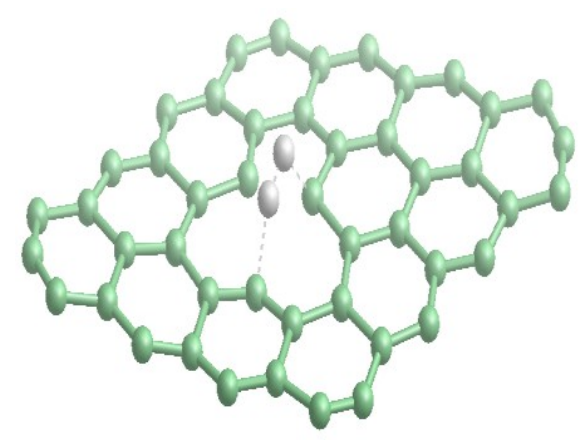

Defective graphenes in the absence of metal can activate in various waysoxygen, hydrogen and other molecules promoting organic reactions. 